\title{
Effects of soil-structure interaction in seismic analysis of buildings with multiple pressurized tuned liquid column dampers
}

\author{
Maurício Vitali Mendes ${ }^{\text {a* }}$ (D) \\ Paulo Marcelo Vieira Ribeiro ${ }^{b}$ \\ Lineu José Pedroso ${ }^{a}$ (iD \\ a Departamento de Engenharia Civil e Ambiental - Universidade de Brasília (UnB), Brasília, DF, Brasil. E-mail: vitali.mendes@gmail.com, \\ lineu@unb.br \\ b Departmento de Engenharia Civil - Universidade Federal de Pernambuco (UFPE), Recife, PE, Brasil. E-mail: paulo.vribeiro@ufpe.br \\ * Corresponding author
}

https://doi.org/10.1590/1679-78255707

\begin{abstract}
In this paper soil-structure interaction (SSI) effects are investigated while an array of Pressurized Tuned Liquid Column Dampers (PTLCD) is employed for seismic vibration control of buildings. This device represents the most general case of a passive damper, with different reduction options to other control devices obtained by simplifying the involved parameters. Soil conditions considerably affect the control device functioning, because dynamic parameters such as natural frequency, damping factor, and natural modes depend on the soil properties. A simplified mathematical model is developed for the building with multiple degrees of freedom connected to a flexible base. For the time-domain analysis, a computational routine is developed for the linearization of the equilibrium equations of the PTLCD, as well as details for the reduction to the other types of passive dampers. Several numerical examples are selected for the analysis of the damper efficiency in reducing seismic vibration considering SSI. These simulations include Kobe earthquake data, which is applied to the model to evaluate the device performance under different scenarios. It is verified the influence of SSI in the natural frequency and structural response, which is related to the earthquake frequency components. Results confirm that the array of PTLCD's can reduce the vibration amplitudes, being more effective for soils with higher stiffness values.
\end{abstract}

\section{Keywords}

Pressurized Tuned Liquid Column Damper, Soil-Structure Interaction, Seismic Analysis, Vibration Control, Buildings

\section{INTRODUCTION}

Numerous technologies have been developed and studied to ensure structural safety and to prevent large vibration amplitudes in engineering structures, such as buildings, towers, and bridges. Control devices are designed with the purpose of damping, isolating and/or acting against vibrations induced by external forces in the structure in order to guarantee its use within project requirements. Passive devices act on the structure without the need for external power supply for its operation. This type of control device absorbs a fraction of the energy from the structure and dissipates it through its motion. In this sense, they are designed to oscillate at the same frequency of the structure, but with a lag, in order to produce an inertia force in the opposite direction to the structural vibration.

Passive control devices are generally defined within the following categories: Tuned Mass Damper (TMD); Tuned Liquid Damper (TLD); Tuned Liquid Column Damper (TLCD). Bigdeli and Kim (2016) conducted experimental studies to 
compare the performance of these different types of passive devices. The individual contributions of mass and damping of each device in the control of seismic induced vibrations were evaluated separately. The TLCD compared to the TLD presents a greater fraction of energy dissipation caused by its damping and, in general, produces a greater reduction in the structural vibration. Comparing the two types of dynamic fluid attenuators, Lee et al. (2011) proposed a new model. A single device that combines the geometry of the TLD with the geometry of the TLCD, wherein one direction the control system behaves solely as the TLD, and perpendicularly works specifically as the TLCD. The authors concluded that the dynamic response of the structure when the excitation is parallel to the part of the device that operates as the TLCD, there is a more significant reduction in the structural vibration. Similar to this described model, Rozas et al. (2016) study numerically and experimentally the control of structure response by a bi-directional tuned liquid column damper (BTLCD). The analysis proves that the device significantly increases the damping of the controlled structure, reducing its displacement and a high decay of the response. The results presented by Souza (2003) point out that the performance of the TLCD is comparable to the other types of liquid attenuators, but with the advantage of using a smaller amount of mass, with more stable behavior, shorter reaction time, and greater flexibility of sizing. Additionaly, Ghosh and Basu (2004); Chakraborty et al. (2012) and Espinoza et al. (2018) verify the efficiency of the TLCD in the control of seismic excitations. The tuned liquid column damper can also ensure operational safety and increase the fatigue life of the structures to which they are coupled as Shahrabi and Bargi (2019) demonstrate through an analytical and numerical study.

Numerous studies have been conducted to improve the efficiency of TLCD in order to minimize the structural response. Gao et al. (1999) found that with an increase in the number of attenuators in the system, there is greater efficiency in the control, however, from a specific number of devices, there are no significant changes. Shum and Xu (2004) investigate the performance of multiple TLCDs for the mitigation of lateral and torsional vibrations of a bridge. The authors note that in order to achieve the maximum reduction of the response in displacements, the ratio between the horizontal length and the total length of the device should be as large as possible. Min et al. (2005) use a building benchmark to analyze the efficiency of multiple TLCD in controlling wind-induced responses. It is verified that for a random stationary process and this structural model, the tuning of the multiple attenuators in the same frequency is more effective than to tune each attenuator in a frequency referring to the different modes of vibration. Sonmez et al. (2016) also evaluate the control of the primary structure under random excitations, including stationary and non-stationary excitations, with the use of TLCDs. However, the authors propose a new semi-active TLCD model (sTLCD), in which the attenuator is connected to the primary structure using an adaptive spring. It is verified that this change gives more robustness to the control system.

To enhance the damper performance, it is possible to combine a closed chamber with pressurized gas to the ends of the TLCD tube. This variation of the TLCD design is commonly referred to as Pressurized Tunned Liquid Column Damper (PTLCD), sealed tuned liquid column damper (STLCD) or tunned liquid column gas damper (TLCGD). The use of pressurized chambers is beneficial for vibration control of structures with high natural frequencies of vibration, as analyzed by Ghosh and Basu (2004). Hochrainer and Ziegler (2006) point out that the addition of this mechanism causes the attenuator performance to increase when compared to the classic TLCD while maintaining its primary qualities. Shum et al. (2008) in a case study of a bridge under wind action explore the possibilities generated by the application of multiple pressurized liquid column attenuators to control the structural response. The presence of the pressurized chambers allows calibration and tuning of the devices at the desired frequencies, with less effort since it is not only dependent on its dimension but also the gas pressure. Hokmabady et al. (2019) studied the structural vibration control of an offshore platform using a hybrid liquid column gas and magneto-rheological damper system. They confirm that the gas pressure is of great importance, which results in a better performance of the whole system, mainly due to limitations of the liquid movement. Bhattacharyya et al. (2017) verify that the pressurized chamber stiffness of the Pressurized Tunned Liquid Column Damper (PTLCD), despite having non-linear behavior, can be expressed by an equivalent linear value for vibration control analysis. However, it is emphasized that by assuming the rigidity of pressurized gas as linear springs, the reduction of the dynamic response of the structure can be slightly overestimated. Regarding the incorporation of the pressurized chambers to the TLCD, it is worth mentioning that it provides great design flexibility for the control device dimensions, and it also facilitates its application in structures subject to excitations with high-frequency components.

It is also essential to highlight the site conditions with regards to soil properties, a factor that can considerably affect the damper efficiency. The fundamental frequency of the structure, to which the device is tuned, can be strongly altered by SSI effects, as discussed by Mendes (2018). When these interaction effects are included, it is necessary to evaluate the SSI interference in the performance of the TLCD for seismic applications. Luco (1998) and Ghosh and Basu (2005) using impedance functions that consider the three systems (soil-structure-damper), analyzed these effects. After studies such as these, in which the influence of SSI on vibration control is evidenced, Mousavi et al. (2013), Farshidianfar and Soheili (2013), and Ozuygur and Gunduz (2018) applied optimization methods to define optimal parameters for the 
passive device connected to a structure subject to seismic action and under the influence of site conditions. Moreover, Jabary and Madabhushi (2018), through experimental analysis of multiple TMDs, evaluated their performance for the dynamic response. Although some papers on PTLCD are being extended for seismic analysis, few studies have been dedicated to the study of these effects concomitantly with SSI. Traditionally structures with vibration dampers have been analyzed using the fixed base model, which does not reflect the influence of the soil characteristics on the system response. As a result, the seismic response of a building with PTLCD for specific site conditions is of great interest to engineering practice.

This work presents an approach for the passive control of seismic induced vibrations in multi-floor buildings, where the effects of soil-structure interaction are applied to the definition of the type of control and the evaluation of the structural dynamic response. Initially, the mathematical model of PTLCD is developed, which is later coupled to a single degree of freedom model with soil-structure interaction. This model is extended to a multi-floor problem, which allows the inclusion of numerous passive devices at the top of the building, tuned according to the natural frequencies of the SSI problem. A generalized feature of the algorithm arises with the reduction of the PTLCD to any other type of passive attenuator (TLCD, TLD, TMD), obtained using a mathematical analogy with control parameters. Computational routines developed with MATLAB software are presented, enabling modal, harmonic, and transient analysis. Kobe earthquake data are applied to a 40-story building where the performance of the control devices is evaluated. The results in free and forced vibrations are in agreement with the simulations developed by Farshidianfar and Soheili (2013) for buildings with SSI in four different types of soil. Numerous complementary simulations are presented, with a study of: i) combining multiple dampers at different natural frequencies of the structure; ii) influence of soil stiffness and damping; iii) pressurizing as a flexibility factor in PTLCD tuning; (iv) linearization of the damping term of the PTLCD. Finally, the influence of soil-structure interaction on the natural frequency and dynamic response of the system is verified. The results confirm that the PTLCD is efficient in reducing displacement amplitudes, being more effective for soils of higher stiffness values. To the authors' knowledge, the proposed procedures, and the generalized mathematical model based on the PTLCD are original and allow an extensive approach for the passive control of seismic vibrations in buildings considering SSI effects.

\section{THEORETICAL FORMULATION}

The formulation for linear dynamic analysis of multiple degrees of freedom structures under seismic excitation is presented in this section, considering SSI at the base of the structure, represented by soil stiffness and damping, and a passive control device at the top of the structure, corresponding to an array of multiples PTLCD (MPTLCD).

\subsection{Pressurized tuned liquid column damper analytical model}

The TLCD, as well as the TMD, operate without the need for external power supply. This type of damper dissipates energy from the structure to which it is coupled by absorbing part of the energy produced in the structure during its vibration. The vibrating energy of the structure is dissipated by the relative movement of the liquid column in relation to the movement of the structure, as emphasized by Souza (2003). In contrast to TMD, which requires additional elements of stiffness and damping, the TLCD uses the gravity force and the energy dissipation of the liquid. These are generated by the movement of the fluid through the head loss positioned inside the attenuator as restoring and viscous forces, respectively.

The typical analytical model of the TLCD, consisting of a U-tube with liquid (usual water) of a constant section where $z(t)$ represents the displacement of the liquid inside the pipe and $x(t)$, the lateral displacement of the structure has the equation of motion expressed by:

$$
\mathrm{m}_{\mathrm{D}} \ddot{\mathrm{z}}(\mathrm{t})+\mathrm{c}_{\mathrm{D}} \dot{\mathrm{z}}(\mathrm{t})+\mathrm{k}_{\mathrm{D}} \mathrm{z}(\mathrm{t})=-\alpha \mathrm{m}_{\mathrm{D}} \ddot{\mathrm{x}}_{\mathrm{g}}(\mathrm{t})
$$

where

$$
\begin{aligned}
& m_{D}=\rho_{f} A L, \alpha=B / L, \\
& c_{D}=\left(\frac{\pi \mathrm{LD} \rho_{\mathrm{f}}}{8}\right) \mathrm{f} \cdot\left|\dot{\mathrm{z}}_{D}\right|+\frac{\rho_{\mathrm{f}} \mathrm{A}}{2}\left(\frac{\mathrm{A}}{A_{O}}-1\right)^{2},
\end{aligned}
$$


$k_{D}=2 \rho_{f} A g$

where $\rho_{\mathrm{f}}$ is the density of water, $A$ and $D$ are the area and diameter of the cross-section of the TLCD, $L$ and $B$ are the total length and length of the horizontal section of the attenuator, $A_{0}$ is the area of the orifice and $g$ is the acceleration of gravity. The term $f$ is expressed as a function of the roughness of the tube $\varepsilon$ and the Reynolds number Re. This coefficient is evaluated with the following expression:

$\frac{1}{\mathrm{f}}=-2 \log \left[\left(\frac{\varepsilon}{3.7 \mathrm{D}}\right)-\frac{5.16}{\operatorname{Re}} \log \left(\frac{\varepsilon}{3.7 \mathrm{D}}+\frac{5.09}{\operatorname{Re}^{0.87}}\right)\right]$

The liquid oscillation frequency $\omega_{D}$ for the TLCD depends on the total length of the liquid column L:

$\omega_{D}=\sqrt{2 g / L}$

Thus, TLCD is distinguished as a long-term system. To solve this problem and increase its applicability, the vertical sections of the U-tube can be sealed and pressurized (Figure 1). The presence of the pressurized chambers allows damper adjustment and tuning at the desired frequency to be set out with less effort, since it is not only dependent on the dimensions of the tube but also on the stiffnes arising in the pressurized cavity.

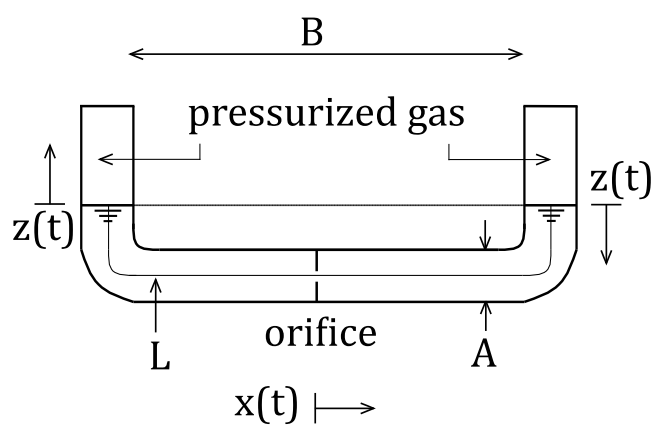

Figure 1. Details of a PTLCD - Sealed vertical edges with two chambers containing pressurized gas.

By implementing compressed air at both ends of the vertical section the Pressurized Tuned Liquid Column Damper (PTLCD) is formed. The PTLCD, when subjected to dynamic loading, presents liquid displacement inside the sealed chambers that, consequently, causes a variation in the pressure of the gas column (Figure 2). In the chamber, P represents the pressure, $V$ its volume, $d V$ the variation of this volume, $d z$ the linear variation of the volume in the direction $z, A$ the area of its cross section and $I_{C}$ the length of the pressurized section.

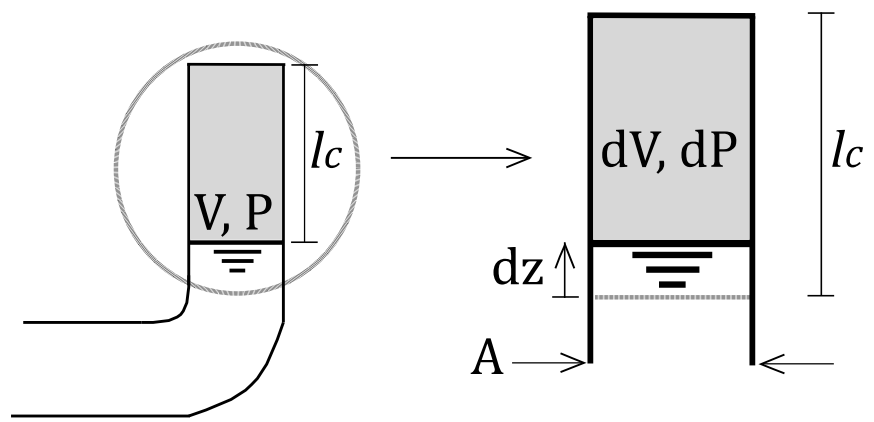

Figure 2 Variation of volume and gas pressure caused by liquid displacement within the chamber.

Considering an isothermal process for the variation of the gas pressure and volume inside the PTLCD chamber:

$\mathrm{P} \cdot \mathrm{V}^{\gamma}=\eta$ 
where $\eta$ is a constant, and $\gamma$ is the gas constant equal to 1.4. Thus, as demonstrated by Pedroso (1992) for the variation of the initial condition inside the cavity:

$\frac{d}{d V}\left(P \cdot V^{\gamma}-\eta\right)=0$

$\frac{d P}{d V} V^{\gamma}+\gamma \cdot P \cdot V^{\gamma-1}=0$

The variation of the gas pressure of the chamber causes a variation in the force expressed by

$d F=d P \cdot A=(-\gamma \cdot P \cdot d V / V) \cdot A$

and considering the linear variation of volume and volume defined by:

$d V=A \cdot d z$ and $V=A \cdot l_{C}$

Results in a force variation dF given by:

$d F=-\gamma \cdot P \cdot A \cdot d z / l_{C}$

Supposing the stiffness of the pressurized cavity $k_{p}$ as a function of the force variation by displacement

$k_{P}=d F / d z$

and with substitution of (10) in (11):

$k_{P}=-\gamma \cdot P \cdot A / l_{C}$

The equation of motion of the PTLCD is given by:

$m_{D} \ddot{z}(t)+c_{D} \dot{z}(t)+\left(k_{D}+2 k_{P}\right) z(t)=-\alpha m_{D} \ddot{x}_{g}(t)$

A comparison of Eqs. (1) and (13) shows that the PTLCD is a more general model when compared to the standard TLCD, with addition of gas cavity stiffness in the two vertical chambers of the device. In a more compact notation:

$m_{D} \ddot{z}(t)+c_{D} \dot{z}(t)+k_{P D} z(t)=-\alpha m_{D} \ddot{x}_{g}(t)$

with:

$k_{P D}=k_{D}+2 k_{P}=2 A\left(\rho_{f} \cdot g+\gamma \cdot P / l_{C}\right)$

\subsection{Soil structure interaction with PTLCD}

During the event of an earthquake, the response of the structure is affected by the interaction of four connected systems: the PTLCD, the structure, the foundation, and the soil. The analysis with soil-structure interaction involves the joint response of these systems (Figure 3). 

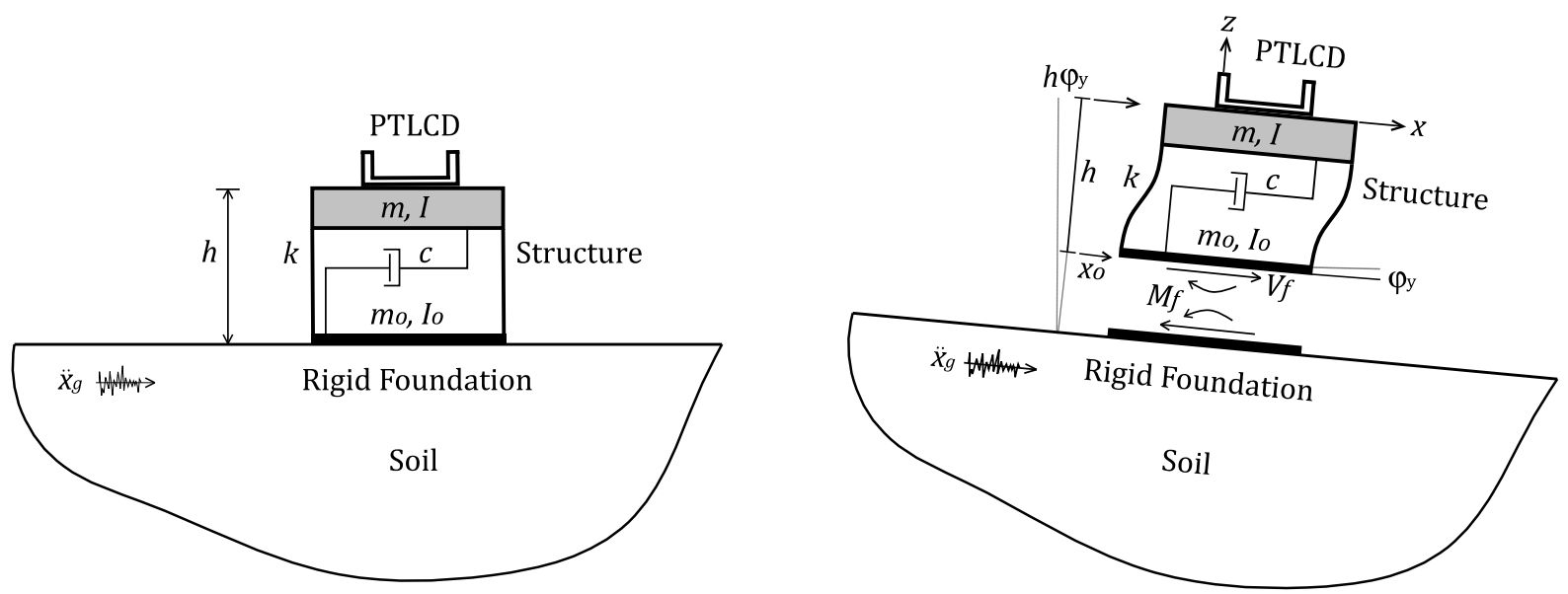

Figure 3 Structure-PTLCD response to ground movement considering SSI.

The free-field acceleration of the soil $\ddot{x}_{\mathrm{g}}(\mathrm{t})$ on the surface of the half-space results in interaction forces at the interface of the structure with its rigid foundation and produces shear force $V_{f}$ and moment $M_{f}$ at the base. These interacting forces unfold displacements $x_{0}(t)$ and rotations $\phi_{y}(t)$ at the soil-foundation interface, together with the displacements of the structure $x(t)$ and the movement of the liquid column $z(t)$ caused by the earthquake.

For the simple case of a structure with a single degree of freedom (SDOF) of height $h$, supported by a foundation of mass $m_{0}$ and mass moment of inertia $I_{0}$, attached to the elastic half-space, the absolute displacement of the structure is expressed by:

$x^{t}(t)=x_{g}(t)+x_{0}(t)+h \varphi_{y}(t)+x(t)$

$x_{g}, x_{0}, \phi_{y}$, and $x$ denote, respectively, the free-field displacement of the soil, horizontal displacement, and rotation of the soil-foundation interface, and the structural relative displacement with respect to foundation in the time domain. From this decomposition, the dynamic equilibrium equations for the movement of the liquid inside the pressurized damper, the translation of the structural mass and the displacements/rotations at the base are established as proposed by Ghosh and Basu (2005).

The equation of motion of the liquid column is given by

$m_{D} \ddot{z}+c_{D} \dot{z}+k_{P D} z=-\alpha m_{D}\left(\ddot{x}_{g}+h \ddot{\varphi}_{y}+\ddot{x}_{0}+\ddot{x}\right)$

and the equation of motion for the structure is

$\left(m+m_{D}\right)\left\{h \ddot{\varphi}_{y}+\ddot{x}_{0}+\ddot{x}\right\}+\alpha m_{D} \ddot{z}+c \dot{x}+k x=-\left(m+m_{D}\right) \ddot{x}_{g}$

where $\mathrm{m}, \mathrm{c}$, and k represent the mass, damping and stiffness of the structure, respectively. For the degrees of freedom of the foundation and elastic half-space interface, the dynamic equation for the translation of the foundation expressed by:

$V_{f}+\left(m+m_{D}\right) \ddot{x}+\left(m_{0}+m+m_{D}\right) \ddot{x}_{0}+\left(m+m_{D}\right) h \ddot{\varphi}_{y}+\alpha m_{D} \ddot{z}=-\left(m_{0}+m+m_{D}\right) \ddot{x}_{g}$

and for the moment $\mathrm{Mf}$ caused by the interaction we have the following equation:

$$
M_{f}+\left(m+m_{D}\right) h\left\{\ddot{x}+\ddot{x}_{0}\right\}+\left\{I_{0}+I+\left(m+m_{D}\right) h^{2}\right\} \ddot{\varphi}_{y}+\alpha m_{D} h \ddot{z}=-\left(m+m_{D}\right) h \ddot{x}_{g}
$$

The interaction forces $V_{f}$ and $M_{f}$ cause displacements and rotations at the soil-foundation interface. Such displacements and rotations are feasible due to the flexibility of the soil-foundation system, which contributes significantly to structural flexibility. The soil dissipates some of the vibration energy of the system, either by wave 
propagation or by the material behavior, considerably affecting the damping of the system. For the scenario of a foundation supported on the soil surface:

$\mathbf{c}_{\mathbf{S}}=\left[\begin{array}{cc}c_{Y Y} & 0 \\ 0 & c_{X}\end{array}\right], \mathbf{k}_{\mathbf{S}}=\left[\begin{array}{cc}k_{Y Y} & 0 \\ 0 & k_{X}\end{array}\right], \mathbf{u}_{\mathbf{s}}{ }^{T}=\left\{\varphi_{y}(t) x_{0}(t)\right\}$

The following linear system arises from the equilibrium of forces of a structure with one degree of freedom, foundation supported under the elastic half-space, and with PTLCD:

$\left[\begin{array}{ccc}\mathbf{m}_{\mathbf{s}} & \mathbf{m}_{\mathrm{SE}} & \mathbf{m}_{\mathrm{SD}} \\ \mathbf{m}_{\mathrm{ES}} & \mathrm{m}+\mathrm{m}_{\mathrm{D}} & \alpha \mathrm{m}_{\mathrm{D}} \\ \mathbf{m}_{\mathrm{DS}} & \alpha \mathrm{m}_{\mathrm{D}} & \mathrm{m}_{\mathrm{D}}\end{array}\right]\left\{\begin{array}{c}\ddot{\mathbf{u}}_{\mathrm{s}} \\ \ddot{\mathrm{x}} \\ \ddot{\mathrm{z}}\end{array}\right\}+\left[\begin{array}{ccc}\mathbf{c}_{\mathrm{s}} & \mathbf{0} & \mathbf{0} \\ \mathbf{0} & \mathrm{c} & 0 \\ \mathbf{0} & 0 & \mathrm{c}_{\mathrm{D}}\end{array}\right]\left\{\begin{array}{c}\dot{\mathrm{u}}_{\mathrm{s}} \\ \dot{\mathrm{x}} \\ \dot{\mathrm{z}}\end{array}\right\}+\left[\begin{array}{ccc}\mathbf{k}_{\mathrm{s}} & \mathbf{0} & \mathbf{0} \\ \mathbf{0} & \mathrm{k} & 0 \\ \mathbf{0} & 0 & k_{P D}\end{array}\right]\left\{\begin{array}{c}\mathbf{u}_{\mathbf{s}} \\ \mathrm{x} \\ \mathrm{z}\end{array}\right\}=\mathbf{P}$

the parameters $\mathrm{m}, \mathrm{c}$ and $\mathrm{k}$ correspond, respectively, to the structural mass, damping and stiffness. The submatrices that form the mass matrix of the system are given by:

$\mathbf{m}_{\mathbf{s}}=\left[\begin{array}{cc}I_{0}+I+\left(m+m_{D}\right) h^{2} & \left(m+m_{D}\right) h \\ \left(m+m_{D}\right) h & m_{0}+m+m_{D}\end{array}\right]$

$\mathbf{m}_{\mathrm{SE}}=\mathbf{m}_{\mathrm{ES}}{ }^{\mathbf{T}}=\left\{\begin{array}{c}\left(m+m_{D}\right) h \\ m+m_{D}\end{array}\right\}, \mathbf{m}_{\mathbf{S D}}=\mathbf{m}_{\mathrm{DS}}{ }^{\mathrm{T}}=\left\{\begin{array}{c}\alpha m_{D} h \\ \alpha m_{D}\end{array}\right\}$

The mass moment of inertia I of each building floor is given by:

$I=\frac{m b^{2}}{12}$

where $b$ is the horizontal dimension of the floor (parallel to seismic excitation direction). The rigid foundation of radius $r$, mass $m_{0}$, and mass moment of inertia $I_{0}$ is supported by homogeneous linear semi-elastic medium. For a circular shallow foundation, the following expression is valid for $I_{0}$ :

$I_{0}=\frac{m_{0} r^{2}}{4}$

The seismic force vector $\boldsymbol{P}$ applied to the structure (representing the excitation caused by ground movement) is defined as a function of the acceleration $\ddot{x}_{\mathrm{g}}(\mathrm{t})$ at the base, and expressed by:

$\boldsymbol{P}^{T}=-\left\{\left(m+m_{D}\right) h \quad m_{0}+m+m_{D} \quad m+m_{D} \quad \alpha m_{D}\right\} \ddot{x}_{g}(t)$

In this work the soil-foundation system is modeled by discrete models proposed by Dobry and Gazetas (1986), although in the computational routine other general parameters for the soil and the foundation can be used, and computed from other mathematical models, e.g.: Pais and Kausel (1988) and Mylonakis et al (2006). The stiffness and damping coefficients of the soil-foundation interaction are determined from simplified models for frequencyindependent impedance functions as presented in Table 1. The equations describe the stiffness and damping for translation $(\mathrm{x})$ and for rotation (yy). 
Table 1 Formulas for stiffness and damping coefficients.

\begin{tabular}{|c|c|c|c|}
\hline$k_{X}(\mathrm{~N} / \mathrm{m})$ & $k_{Y Y}(\mathrm{~N} \cdot \mathrm{m})$ & $c_{X}(\mathrm{~N} \cdot \mathrm{s} / \mathrm{m})$ & $c_{Y Y}(\mathrm{~N} \cdot \mathrm{s} \cdot \mathrm{m})$ \\
\hline $8 \cdot G_{S} \cdot r$ & $8 \cdot G_{S} \cdot r^{3}$ & $4.6 \cdot \rho_{S} \cdot V_{S} \cdot r^{2}$ & $0.4 \cdot \rho_{S} \cdot V_{S} \cdot r^{4}$ \\
\hline $2-\nu$ & $\overline{3(1-\nu)}$ & $2-\nu$ & $1-\nu$ \\
\hline
\end{tabular}

The soil shear modulus, $G_{s}$, is determined from its modulus of elasticity, $E_{s}$, and Poisson's coefficient, $v_{s}$, while the shear wave velocity $V_{S}$ is given as a function of the shear modulus and the specific mass of the soil:

$$
G_{S}=\frac{E_{S}}{2\left(1+\nu_{s}\right)}, V_{S}=\sqrt{\frac{G_{S}}{\rho_{s}}}
$$

\subsection{Generalization for multiple degrees of freedom}

For the scenario of a multi-floor building with multiple PTLCDs (MPTLCD) coupled to the last floor, as shown in Figure 4, the dynamic equilibrium matrix equation has its matrices formed of submatrices referring to each of the systems and to the interaction between the different systems as is expressed in Equation (29).
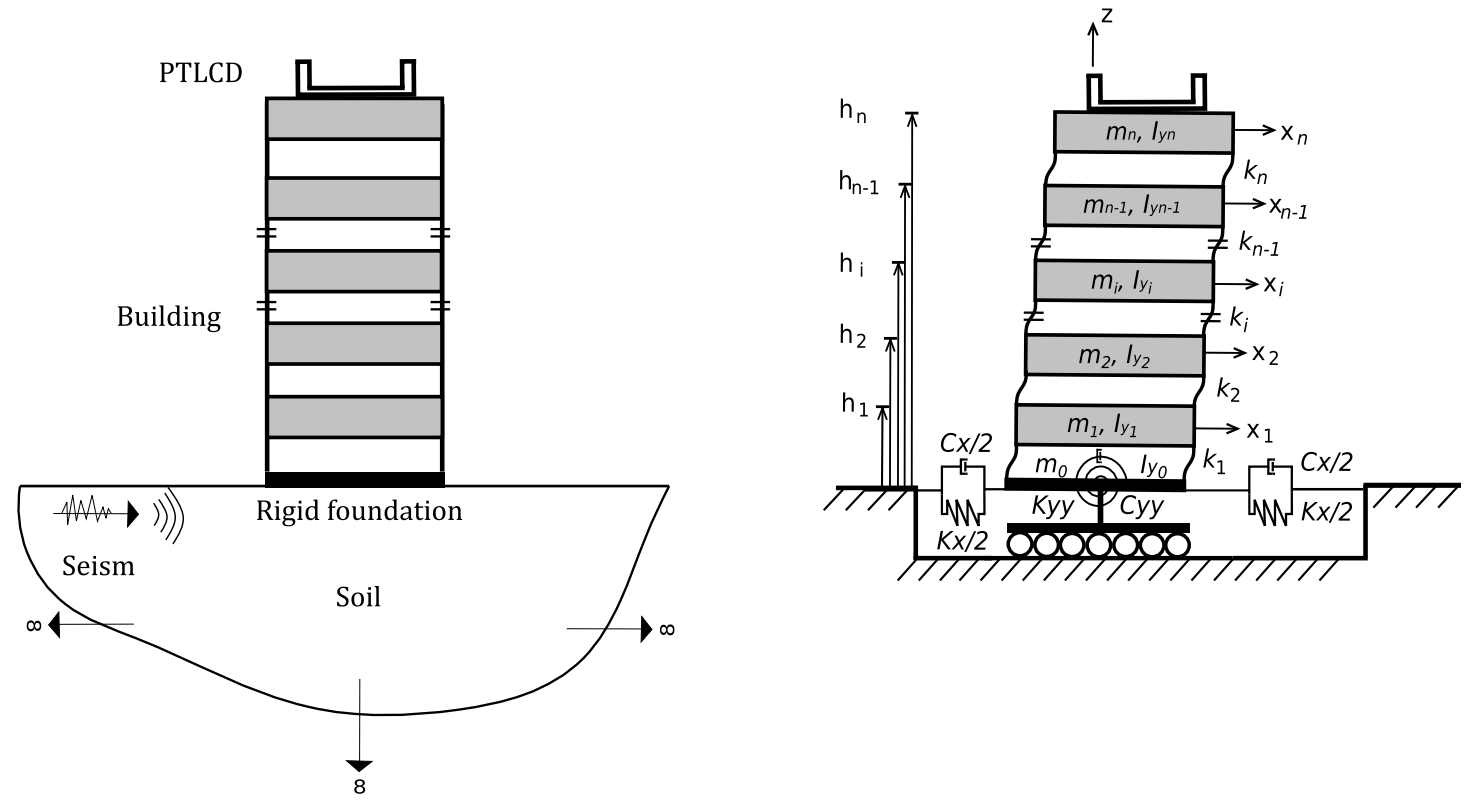

Figure 4 MDOF structure-PTLCD system considering SSI.

$\left[\begin{array}{ccc}\mathbf{m}_{\mathrm{S}} & \mathbf{m}_{\mathrm{SE}} & \mathbf{m}_{\mathrm{SD}} \\ \mathbf{m}_{\mathrm{ES}} & \mathbf{m}_{\mathrm{E}} & \mathbf{m}_{\mathrm{ED}} \\ \mathbf{m}_{\mathrm{DS}} & \mathbf{m}_{\mathrm{DE}} & \mathbf{m}_{\mathrm{D}}\end{array}\right]\left\{\begin{array}{l}\ddot{\mathbf{u}}_{\mathrm{S}} \\ \ddot{\mathbf{u}}_{\mathrm{E}} \\ \ddot{\mathbf{u}}_{\mathrm{D}}\end{array}\right\}+\left[\begin{array}{ccc}\mathbf{c}_{\mathrm{S}} & \mathbf{0} & \mathbf{0} \\ \mathbf{0} & \mathbf{c}_{\mathrm{E}} & \mathbf{0} \\ \mathbf{0} & \mathbf{0} & \mathbf{c}_{\mathrm{D}}\end{array}\right]\left[\begin{array}{c}\dot{\mathbf{u}}_{\mathrm{S}} \\ \dot{\mathbf{u}}_{\mathrm{E}} \\ \dot{\mathbf{u}}_{\mathrm{D}}\end{array}\right]+\left[\begin{array}{ccc}\mathbf{k}_{\mathrm{S}} & \mathbf{0} & \mathbf{0} \\ \mathbf{0} & \mathbf{k}_{\mathrm{E}} & \mathbf{0} \\ \mathbf{0} & \mathbf{0} & \mathbf{k}_{\mathrm{D}}\end{array}\right]\left\{\begin{array}{l}\mathbf{u}_{\mathrm{S}} \\ \mathbf{u}_{\mathrm{E}} \\ \mathbf{u}_{\mathrm{D}}\end{array}\right\}=-\mathbf{P}$

The mass matrix of the system comprises submatrices referring to individual systems and by submatrices that represent the coupling effect between these different systems. The submatrix $m_{E}$ defines the lumped mass matrix of the $\mathrm{n}$ floor structure, and also includes the contribution of the MPTLCDs masses present on the last floor: 
$\mathbf{m}_{\mathbf{E}}=\left[\begin{array}{ccccc}m_{1} & 0 & \cdots & 0 \\ 0 & m_{2} & & & \vdots \\ \vdots & & \ddots & & 0 \\ & & & m_{n-1} & m_{n}+\sum_{j=1}^{q} m_{D j}\end{array}\right]$

Considering a quantity $q$ of PTLCDs the mass matrix $m_{D}$ and the submatrix of mass $m_{E D}$ are expressed by:

$\mathbf{m}_{\mathbf{D}}=\left[\begin{array}{cccc}\mathrm{m}_{\mathrm{D}_{1}} & 0 & \cdots & 0 \\ 0 & \mathrm{~m}_{\mathrm{D}_{2}} & & \vdots \\ \vdots & & \ddots & 0 \\ 0 & \cdots & 0 & \mathrm{~m}_{\mathrm{D}_{\mathrm{q}}}\end{array}\right], \mathbf{m}_{\mathrm{DE}}=\mathbf{m}_{\mathrm{ED}}{ }^{\mathrm{T}}=\left[\begin{array}{cccc}0 & \cdots & 0 & \alpha m_{D_{1}} \\ 0 & \cdots & 0 & \alpha m_{D_{2}} \\ \vdots & & \vdots & \vdots \\ 0 & \cdots & 0 & \alpha m_{D_{q}}\end{array}\right]$

The mass submatrices referring to the soil-structure interaction are given by:

$\mathbf{m}_{\mathbf{S}}=\left[\begin{array}{cc}I_{0}+\sum_{i=1}^{n}\left(I_{i}+m_{i} h_{i}^{2}\right)+\sum_{j=1}^{q} m_{D j} h_{n}^{2} & \sum_{i=1}^{n} m_{i} h_{i}+\sum_{j=1}^{q} m_{D_{j}} h_{n} \\ \sum_{i=1}^{n} m_{i} h_{i}+\sum_{j=1}^{q} m_{D_{j}} h_{n} & m_{0}+\sum_{i=1}^{n} m_{i}+\sum_{j=1}^{q} m_{D_{j}}\end{array}\right]$

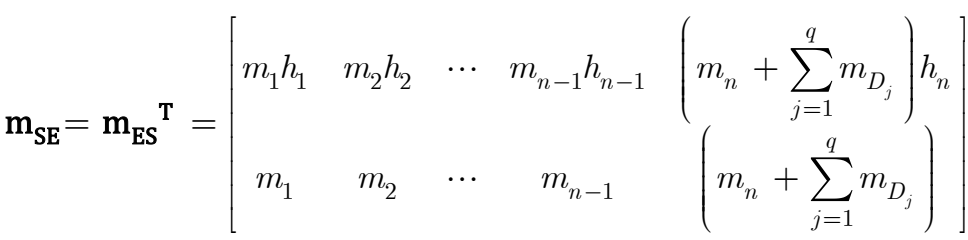

$\mathbf{m}_{\mathbf{S D}}=\mathbf{m}_{\mathrm{DS}}{ }^{\mathbf{T}}=\left[\begin{array}{ccc}\alpha m_{D_{1}} h_{n} & \cdots & \alpha m_{D_{q}} h_{n} \\ \alpha m_{D_{1}} & \cdots & \alpha m_{D_{q}}\end{array}\right]$

The stiffness and damping matrix do not present coupling between the different systems, and their formulations are unchanged, as presented in the matrix equation of a SDOF structure. The foundation stiffness sub-matrix $\mathbf{k}_{\mathbf{s}}$ and damping $c_{s}$ follow the same formulation presented in Eq. (21), while the stiffness matrix of the n-structure DOF and the matrix of dampers $\mathbf{k}_{\mathbf{D}}$ with q PTLCDs are respectively defined in expression Eq. (35).

$\mathbf{k}_{\mathbf{E}}=\left[\begin{array}{ccccc}k_{1}+k_{2} & -k_{2} & 0 & \cdots & 0 \\ -k_{2} & k_{2}+k_{3} & -k_{3} & & \vdots \\ 0 & -k_{3} & \ddots & -k_{n-1} & 0 \\ \vdots & & -k_{n-1} & k_{n-1}+k_{n} & -k_{n} \\ 0 & \cdots & 0 & -k_{n} & k_{n}\end{array}\right], \mathbf{k}_{\mathbf{D}}=\left[\begin{array}{cccc}k_{P D_{1}} & 0 & \cdots & 0 \\ 0 & k_{P D_{2}} & & \vdots \\ \vdots & & \ddots & 0 \\ 0 & \cdots & 0 & k_{P D_{q}}\end{array}\right]$

The structural damping matrix $\mathbf{C}_{\mathbf{E}}$ is determined by the Rayleigh Damping:

$\mathbf{c}_{\mathbf{E}}=A_{0} \mathbf{m}_{\mathbf{E}}+A_{1} \mathbf{k}_{\mathbf{E}}$ 
with:

$$
\mathrm{A}_{0}=2 \xi \frac{\omega_{1} \cdot \omega_{\mathrm{n}}}{\omega_{1}+\omega_{\mathrm{n}}} \mathrm{A}_{1}=\frac{2 \xi}{\omega_{1}+\omega_{\mathrm{n}}}
$$

$\omega_{1}$ and $\omega_{n}$ are equivalent to the first and last natural undamped frequency of the building, and $\xi$ is the damping ratio.

The MPTLCD damping submatrix $C_{D}$ is given by

$$
\mathbf{c}_{\mathbf{D}}=\left[\begin{array}{cccc}
c_{D_{1}} & 0 & \cdots & 0 \\
0 & c_{D_{2}} & & \vdots \\
\vdots & & \ddots & 0 \\
0 & \cdots & 0 & c_{D_{q}}
\end{array}\right]
$$

The vector force $\mathbf{P}$ for the case of the acceleration applied at the base of the system coupled with the load as a function of the acceleration at the base, $\ddot{x}_{g}(t)$, is defined as

$$
\boldsymbol{P}^{T}=\left\{\sum_{i=1}^{n} m_{i} h_{i}+h_{n} \sum_{j=1}^{q} m_{D_{j}} \quad m_{0}+\sum_{i=1}^{n} m_{i}+\sum_{j=1}^{q} m_{D_{j}} m_{1} \quad \cdots \quad m_{n}+\sum_{j=1}^{q} m_{D_{j}} \alpha m_{D_{1}} \quad \cdots \quad \alpha m_{D_{q}}\right\} \cdot \ddot{x}_{g}
$$

while the dynamic response $\mathbf{u}$ of the system is given by:

$$
\boldsymbol{u}^{\mathrm{T}}=\left\{\begin{array}{llllllllll}
\varphi_{y}(t) & x_{0}(t) & x_{1}(t) & x_{2}(t) & \cdots & x_{n-1}(t) & x_{n}(t) & z_{1}(t) & \cdots & z_{q}(t)
\end{array}\right\}
$$

\section{COMPUTATIONAL ASPECTS}

An algorithm was developed in MATLAB for the dynamic analysis of the SSI-MPTLCD systems, and it is available in GitHub. The code performs modal analysis, and transient dynamic analysis in both time and frequency domain (harmonic loading for a range of excitation frequencies).

It is possible to vary the quantity of PTLCDs and their features (dimensions), as well as the presence or absence of the pressurized chamber (simulating TLCD). Also, the submatrices containing the control device parameters can be easily adapted and extended to the TMD and TLD cases, where equivalent parameters can be considered for these control devices. An additional feature is the option of fixed or flexible base for the SSI. For the SSI model, it is possible to define different flexibility and damping coefficients for each type of soil, as well as the mass and the mass moment of inertia of the foundation.

The numerical integration of equilibrium equations is conceived with an adjustment in the method of constant average acceleration presented by Chopra (1995) apud Newmark (1959): a linearization procedure is applied to the damper equation at each time step.

To evaluate the increment in the displacement $\Delta \mathbf{u}_{i}$ of the system, given by:

$$
\Delta \mathbf{u}_{i}=\hat{\mathbf{k}}_{i}^{-1} \Delta \hat{\mathbf{P}}_{i}
$$

it is necessary to determine $\hat{\mathbf{k}}_{\mathrm{i}}$ and $\Delta \widehat{\mathbf{P}}_{\mathrm{i}}$ for each time step $\Delta \mathrm{t}$, since both assume different values throughout the integration, and the damping matrix of the system $\mathbf{c}_{i}$ and the damping values of the PTLCD $C_{D j}$, in turn, depend on the liquid velocity inside the tube (Eq. $2 b$ ). Thus, at each time step, a new damping coefficient $C_{D j}$ is defined and the damping matrix of system $\mathbf{c}_{i}$ is updated and then $\hat{\mathbf{k}}_{i}$ and $\Delta \widehat{\mathbf{P}}_{i}$ are computed with:

$\hat{\mathbf{k}}_{i}=\mathbf{k}+\frac{\gamma}{\beta \Delta \mathrm{t}} \mathbf{c}_{i}+\frac{1}{\beta(\Delta \mathrm{t})^{2}} \mathbf{m}$ 
$\Delta \hat{\mathbf{P}}_{i}=\Delta \mathbf{P}_{i}+\left(\frac{1}{\beta \Delta \mathrm{t}} \mathbf{m}+\frac{\gamma}{\beta} \mathbf{c}_{i}\right) \dot{\mathbf{u}}_{i}+\left[\frac{1}{2 \beta} \mathbf{m}+\left(\frac{\gamma \Delta \mathrm{t}}{\beta}-\Delta \mathrm{t}\right) \mathbf{c}_{i}\right] \ddot{\mathbf{u}}_{i}$

where $\Delta \mathbf{P}=\mathbf{P}_{(i+1)}+\mathbf{P}_{\mathrm{i}}$. Subsequently, the increase in velocity $\Delta \dot{\mathbf{u}}_{\mathrm{i}}$ and the increase in acceleration $\Delta \ddot{\mathbf{u}}_{\mathrm{i}}$ are defined by:

$$
\begin{aligned}
& \Delta \dot{\mathbf{u}}_{i}=\frac{\gamma}{\beta \Delta \mathrm{t}} \Delta \mathbf{u}_{i}-\frac{\gamma}{\beta} \dot{\mathbf{u}}_{i}+\Delta \mathrm{t}\left(1-\frac{\gamma}{2 \beta}\right) \ddot{\mathbf{u}}_{i} \\
& \Delta \ddot{\mathbf{u}}_{i}=\frac{1}{\beta \Delta \mathrm{t}^{2}} \Delta \mathbf{u}_{i}-\frac{1}{\beta \Delta \mathrm{t}} \dot{\mathbf{u}}_{i}-\frac{1}{2 \beta} \ddot{\mathbf{u}}_{i}
\end{aligned}
$$

A general algorithm with each step of the computational routine described above is summarized in Table 2.

Table 2 Algorithm for the evaluation of the dynamic response of the SSI system with MPTLCD.

1. Data input.

2. Data output: dynamic system response.

3. Initial calculations.

3.1. Establish the matrices of the system: $\mathbf{m}, \mathbf{c}, \mathbf{k}, \mathbf{P}$ (Eqs. 29-40).

3.2. Initial acceleration: $\ddot{\mathbf{u}}_{0}=\mathbf{m}^{-1}\left(\mathbf{P}_{0}-\mathbf{c} \dot{\mathbf{u}}_{0}-\mathrm{k} \mathbf{u}_{0}\right)$.

3.3. Define $\Delta t$ and the integration constants $\beta$ and $\gamma$

4. Computation for each time step, $i=1,2,3 \ldots$

4.1. PTLCD damping ( $\left.C_{D j}\right)$ defined for step $i$ (Eq. $\left.2 b\right)$.

4.2. Set matrix $\mathbf{c}_{\mathbf{i}}$.

4.3. Compute $\hat{\mathbf{k}}_{\mathrm{i}}$ (Eq. 42) and $\Delta \hat{\mathbf{P}}_{\mathrm{i}}$ (Eq. 43).

4.4. Evaluate $\Delta \mathbf{u}_{\mathrm{i}}, \Delta \dot{\mathbf{u}}_{\mathrm{i}}$ and $\Delta \ddot{\mathbf{u}}_{\mathrm{i}}$ (Eqs. 41, 44 and 45).

4.5. $\mathbf{u}_{\mathrm{i}+1}=\mathbf{u}_{\mathrm{i}}+\Delta \mathbf{u}_{\mathrm{i}}, \dot{\mathbf{u}}_{\mathrm{i}+1}=\dot{\mathbf{u}}_{\mathrm{i}}+\Delta \dot{\mathbf{u}}_{\mathrm{i}}$ and $\ddot{\mathbf{u}}_{\mathrm{i}+1}=\ddot{\mathbf{u}}_{\mathrm{i}}+\Delta \ddot{\mathbf{u}}_{\mathrm{i}}$.

5. Repeat for the next step. Change $i$ to $i+1$ and return to step 4 .

\subsection{Equivalent parameters for other types of passive control devices}

The generalized aspect of the computational routine arises with the reduction of the PTLCD or any other type of passive damper (TLCD, TLD, and TMD) by setting equivalent parameters of the control device by means of a mathematical analogy.

Considering a SDOF structure, where $\mathrm{m}, \mathrm{c}$, and k define the structural mass, damping, and stiffness, respectively, with a coupled passive device, the dynamic equilibrium equation of the system is expressed as:

$\left[\begin{array}{cc}\left(m+m_{e q}\right) & m^{*} \\ m^{*} & m_{e q}\end{array}\right]\left\{\begin{array}{l}\ddot{x} \\ \ddot{z}\end{array}\right\}+\left[\begin{array}{cc}c & 0 \\ 0 & c_{e q}\end{array}\right]\left\{\begin{array}{c}\dot{x} \\ \dot{z}\end{array}\right\}+\left[\begin{array}{cc}k & 0 \\ 0 & k_{e q}\end{array}\right]\left\{\begin{array}{c}x \\ z\end{array}\right\}=-\left\{\begin{array}{c}\left(m+m_{e q}\right) \\ m^{*}\end{array}\right\} \ddot{x}_{g}$

where $m_{e q}, m^{*}, c_{e q}$ and $k_{e q}$ denote to the equivalent mass, coupled mass, damping and stiffness parameters of the passive damper (TLCD, TLD or TMD), with the dynamic response of the device defined by $z$ and its derivatives.

Equation (46) establishes a mass coupling between the structure and the passive device. For the TMD the use of the equivalent parameters is straightforward, once the optimal parameters of the attenuator are determined, either computationally as Marano et al. (2010) or from classic solutions proposed by Hartog (1956), Warbuton (1981) and Warbuton (1982). Equivalent parameters of the TLD are discussed by Banerji et al. (2000), Souza (2003), Bigdeli and Kim (2016), and Tait (2008). Thus, once the equivalent parameters of the passive device are established, it is enough to proceed with the solution method for Eq. (46) to determine the dynamic response of the structure-damper system. 


\section{NUMERICAL EXAMPLES}

This section presents the dynamic responses of MDOF buildings with pressurized dampers tuned to different frequencies of the structure on different soil types. The efficiency in reducing structural oscillations considering SSI under Kobe earthquake excitation, obtained from the data provided by the Center for Engineering Strong Motion Data (CESMD, 2018), is investigated through a parametric study in terms of mass ratio, damper frequency and soil properties

\subsection{Effects of the soil-structure interaction on the coupled system natural frequency}

In order to examine the effects of SSI on buildings, the model of shear building and soil adopted by Liu et al. (2008) and later by Farshidianfar and Soheili (2013) for the analysis of wind and earthquake vibrations, respectively, is employed. The structural properties are presented in Table 3. The flexible base of the model is determined by relating to three soil types with different mechanical parameters which, are defined as dense, medium and soft soil. Table 4 indicates the values of the stiffness and damping coefficients adopted for the springs and dashpots positioned at the base of the model for each soil type.

Table 3 Structural model data (adapted from Farshidianfar and Soheili (2013))

\begin{tabular}{cc}
\hline No. of stories & 40 \\
\hline Story height $\left(h_{i}\right)$ & $4 \mathrm{~m}$ \\
Story mass $\left(\mathrm{m}_{\mathrm{i}}\right)$ & $9.8 \cdot 10^{5} \mathrm{~kg}$ \\
Story moment of inertia $\left(\mathrm{l}_{\mathrm{i}}\right)$ & $1.31 \cdot 10^{8} \mathrm{~kg} \cdot \mathrm{m}^{2}$ \\
Story stiffness $\left(\mathrm{k}_{\mathrm{i}}\right)$ & $\mathrm{K} 1=2.13 \cdot 10^{9} \mathrm{~N} / \mathrm{m}$ \\
& $\mathrm{K} 40=9.98 \cdot 10^{8} \mathrm{~N} / \mathrm{m}$ \\
Story stiffness linear decay $\left(\mathrm{k}_{\mathrm{i}}\right)$ & $2.87 \cdot 10^{7} \mathrm{~N} / \mathrm{m}$ \\
Structural damping $(\xi)$ & $3.43 \%$ \\
Foundation radius $(\mathrm{r})$ & $20 \mathrm{~m}$ \\
Foundation mass $\left(\mathrm{m}_{0}\right)$ & $1.96 \cdot 10^{6} \mathrm{~kg}$ \\
Foundation moment of inertia $\left(\mathrm{l}_{0}\right)$ & $1.96 \cdot 10^{8} \mathrm{~kg} \cdot \mathrm{m}^{2}$ \\
\hline
\end{tabular}

Table 4 Soil parameters and flexibility and damping coefficients.

\begin{tabular}{|c|c|c|c|c|c|c|c|c|}
\hline Soil & $\mathbf{N}$ & $\rho_{s}\left(\mathrm{~kg} / \mathrm{m}^{3}\right)$ & $V_{s}(m / s)$ & $\mathrm{G}_{\mathrm{s}}\left(\mathrm{N} / \mathrm{m}^{2}\right)$ & $\mathrm{K}_{\mathrm{X}}(\mathrm{N} / \mathrm{m})$ & $\mathrm{K}_{\mathrm{YY}}(\mathrm{N} \cdot \mathrm{m})$ & $\mathrm{C}_{\mathrm{X}}(\mathrm{N} \cdot \mathrm{s} / \mathrm{m})$ & $\mathrm{C}_{\mathrm{YY}}(\mathrm{N} \cdot \mathrm{s} \cdot \mathrm{m})$ \\
\hline Dense & 0.33 & 2400 & 500 & $6.00 \cdot 10^{8}$ & $5.75 \cdot 10^{10}$ & $1.91 \cdot 10^{13}$ & $1.32 \cdot 10^{9}$ & $1.15 \cdot 10^{11}$ \\
\hline Medium & 0.48 & 1900 & 300 & $1.71 \cdot 10^{8}$ & $1.80 \cdot 10^{10}$ & $7.02 \cdot 10^{12}$ & $6.90 \cdot 10^{8}$ & $7.02 \cdot 10^{10}$ \\
\hline Soft & 0.49 & 1800 & 100 & $1.80 \cdot 10^{7}$ & $1.91 \cdot 10^{9}$ & $7.53 \cdot 10^{11}$ & $2.19 \cdot 10^{8}$ & $2.26 \cdot 10^{10}$ \\
\hline
\end{tabular}

The values obtained by the algorithm implemented in MATLAB in comparison with the natural frequencies of the fixed base and flexible base are presented in Table 5. The results with asterisk represent the values obtained by Farshidianfar and Soheili (2013).

Table 5 Undamped natural frequencies of the system.

\begin{tabular}{cccccccccc}
\hline Model & $\omega_{1}{ }^{*}(\mathrm{rad} / \mathrm{s})$ & $\omega_{1}(\mathrm{rad} / \mathrm{s})$ & Rel. Diff. (\%) & $\omega_{\mathbf{2}}{ }^{*}(\mathrm{rad} / \mathrm{s})$ & $\omega_{\mathbf{2}}(\mathrm{rad} / \mathrm{s})$ & Rel. Diff. (\%) & $\omega_{\mathbf{3}}{ }^{*}(\mathrm{rad} / \mathrm{s})$ & $\omega_{\mathbf{3}}(\mathrm{rad} / \mathrm{s})$ & Rel. Diff. (\%) \\
\hline Fixed base & 1.65 & 1.65 & 0.00 & 4.60 & 4.60 & 0.00 & 7.60 & 7.61 & 0.13 \\
Dense soil & 1.61 & 1.60 & 0.63 & 4.59 & 4.59 & 0.00 & 7.59 & 7.60 & 0.13 \\
Medium soil & 1.54 & 1.54 & 0.00 & 4.58 & 4.58 & 0.00 & 7.58 & 7.59 & 0.13 \\
Soft soil & 1.09 & 1.08 & 0.93 & 4.44 & 4.44 & 0.00 & 7.40 & 7.40 & 0.00 \\
\hline
\end{tabular}

The maximum response of each system as a function of the excitation frequency is presented in Figures 5-7, where the peaks represent the natural frequency values of the structure and are in agreement with the values shown in Table 5. $R_{40}$ corresponds to the ratio between the relative displacement values for the $40^{\text {th }}$ floor, defined in Eq. (16), and the sinuidal acceleration amplitude at the base. By means of the graphical representation of the forced response of the system (Table 3 and 4), it can be noted that the fundamental frequency of the system, when compared to a fixed base value, is greatly reduced under a SSI hypothesis. These plots also provide a greater insight to the dynamic response, since 
it becomes clear how the structural response is affected by soils with different features and are complementary to the eigenvector analysis.

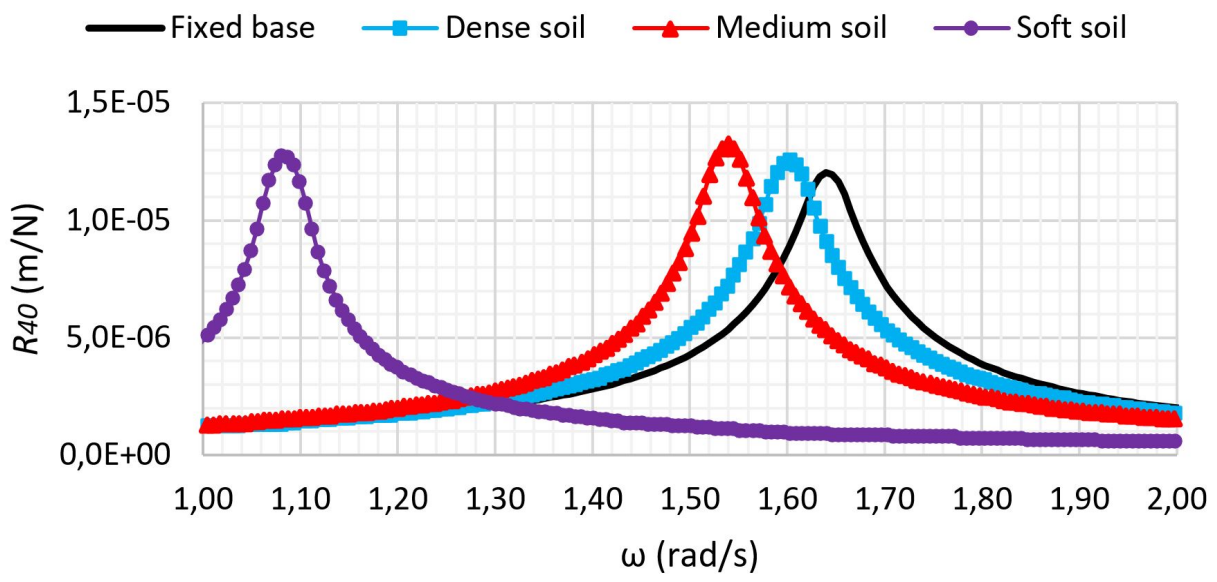

Figure 5 Structure $40^{\text {th }}$ story response on fixed base and with SSI for $1^{\text {st }}$ mode.

$\longrightarrow$ Fixed base $\longrightarrow$-Dense soil $\longrightarrow$ Medium soil $\longrightarrow$ - Soft soil

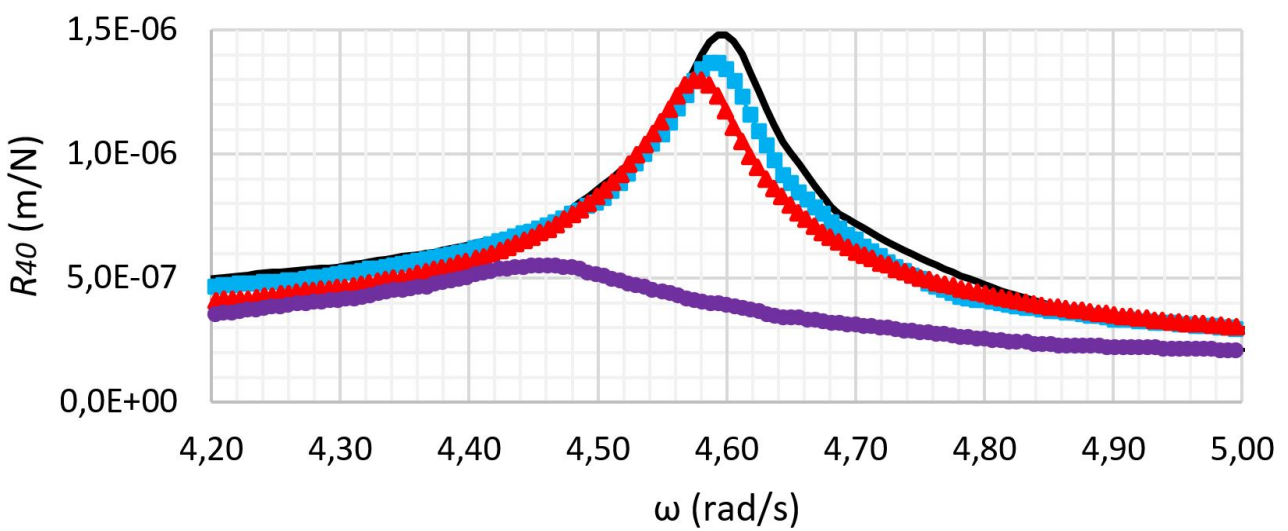

Figure 6 Structure $40^{\text {th }}$ story response on fixed base and with SSI for 2 nd mode.

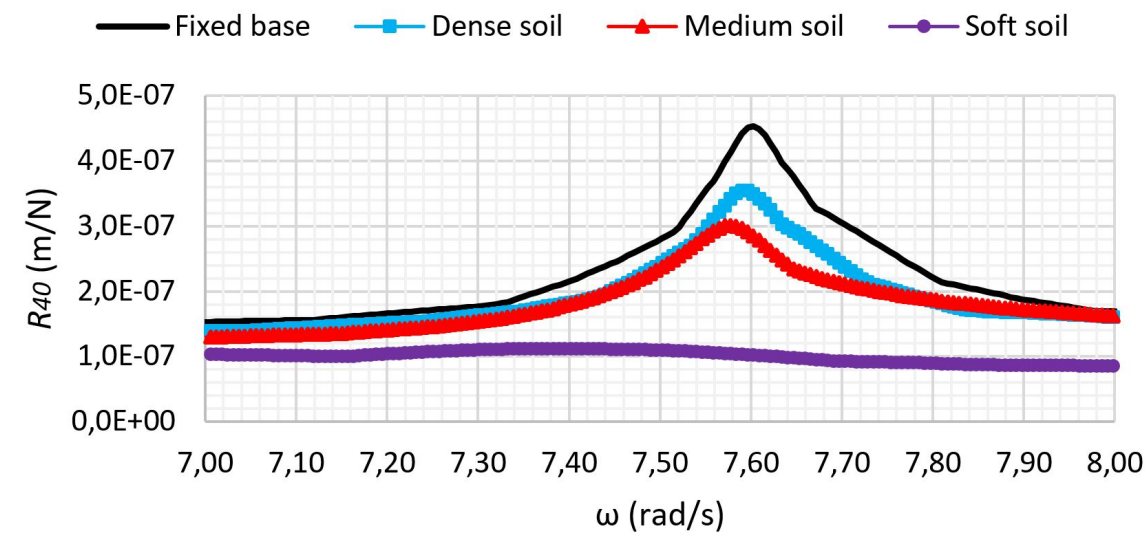

Figure 7 Structure 40th story response on fixed base and with SSI for 3rd mode.

\subsection{Effects of the soil-structure interaction on the system forced response}

The system response under forced vibration is verified using the ground acceleration history recorded in Takatori station, Japan, during the 6.7 magnitude earthquake occurred on January 16, 1995, in Kobe. Figure 8 presents the accelerogram and the frequency spectrum calculated from the algorithm set forth in Aghayan et al. (2016) for this earthquake. 
a)

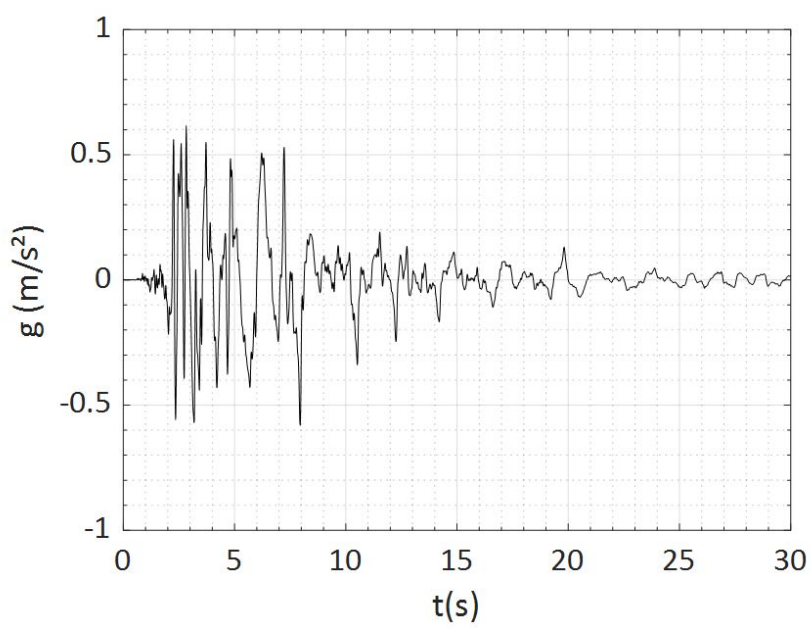

b)

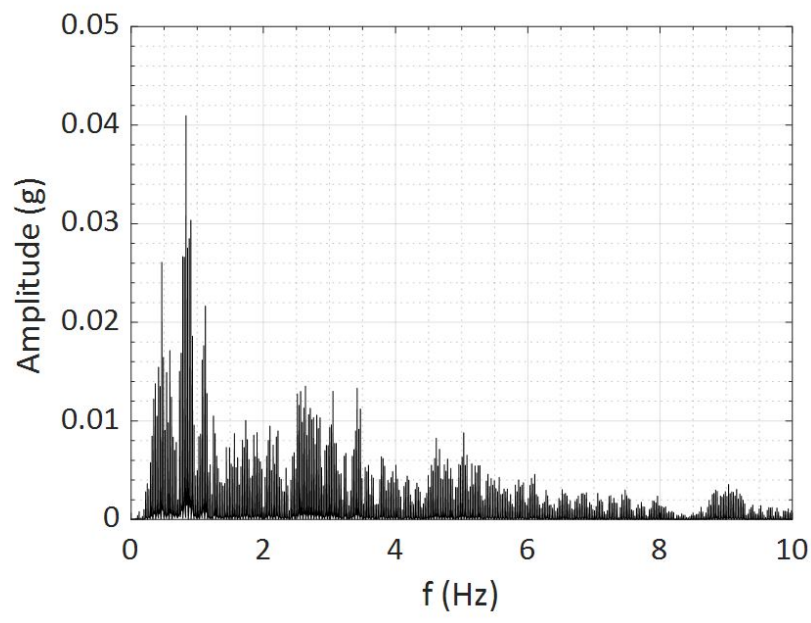

Figure 8 Kobe earthquake: a) acceleration spectrum; b) frequency spectrum.

Table 6 and Figure 9 present the absolute displacement values for the $40^{\text {th }}$ floor produced by Kobe earthquake excitation, and compared to the results obtained by Farshidianfar and Soheili (2013). Table 6 indicates nearly identical results with relative differences inferior to $0.5 \%$.

Table 6 Maximum absolute displacements of the structure (40th floor) under Kobe earthquake excitation.

\begin{tabular}{ccccc}
\hline Model & Fixed base & Dense soil & Medium soil \\
$\mathbf{x}_{\mathbf{4 0}}(\mathbf{m})$ & $\begin{array}{c}\text { Soft soil } \\
\mathbf{x}_{\mathbf{4 0}}(\mathbf{m})\end{array}$ & 1.0615 & 0.7588 \\
\hline Farshidianfar e Soheili (2013) & 1.0459 & 1.0576 & 1.0665 & 0.7566 \\
Algorithm & 1.0461 & 1.0592 & 0.4710 & 0.2899 \\
Relative difference (\%) & 0.0191 & 0.1513 & 0 \\
\hline
\end{tabular}

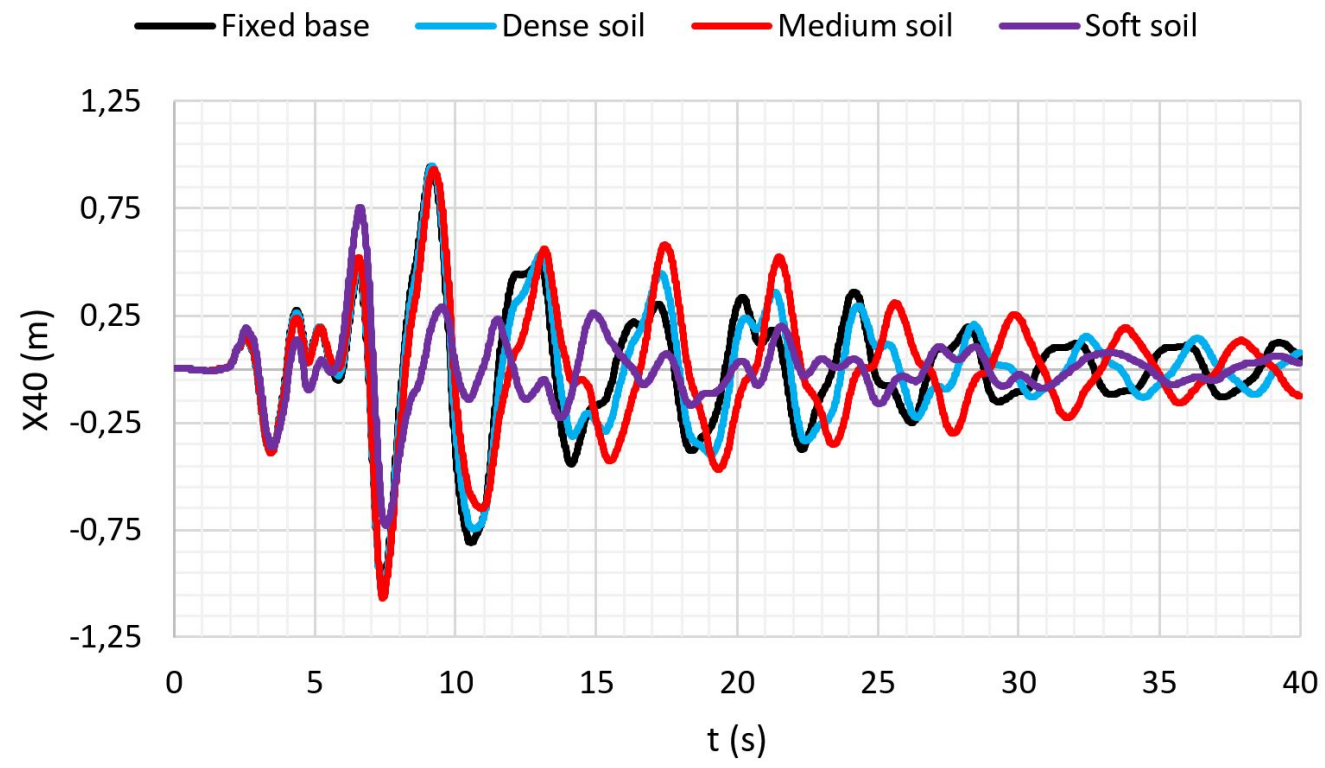

Figure 9 Absolute displacements of the structure $\left(40^{\text {th }}\right.$ floor $)$ under Kobe earthquake excitation.

The structural response for each type of soil is also examined in terms of relative displacements of the structure with respect to the foundation (Table 7). Initially SSI considering only soil flexibility influence $\left(x^{1}\right)$, then the effect of adding soil damping $\left(\mathrm{x}^{2}\right)$. Analysis of the maximum and rms (roots mean square) relative displacements during a frame of 40 seconds of the structure $40^{\text {th }}$ floor, indicate that relative displacements are greatly reduced for the soft soil type, and that damping effects are almost negligible for the analyzed scenarios. 
Table 7 Influence of soil flexibility and soil damping on the structure relative displacements ( $40^{\text {th }}$ floor).

\begin{tabular}{|c|c|c|c|c|c|c|}
\hline Soil & $\operatorname{xmax}^{1}(m)$ & $\operatorname{xmax}^{2}(\mathrm{~m})$ & Rel. Diff. (\%) & $\operatorname{xrms}^{1}(m)$ & $\operatorname{xrms}^{2}(\mathrm{~m})$ & Rel. Diff. (\%) \\
\hline Dense & 1.0132 & 1.0124 & 0.0790 & 0.2701 & 0.2694 & 0.2592 \\
\hline Medium & 0.9519 & 0.9484 & 0.3677 & 0.2562 & 0.2532 & 1.1710 \\
\hline Soft & 0.4497 & 0.4340 & 3.4912 & 0.0846 & 0.0805 & 4.8351 \\
\hline
\end{tabular}

\subsection{Analysis of multiples PTLCD for fixed base structure}

The use of TLCD for control of structural vibrations of the building with parameters presented in Table 3 is possible only with the addition of the pressurized chamber to the device, i.e. using PTLCD. The TLCD, when tuned to the fundamental frequency of the structure under the fixed base scenario $(1.65 \mathrm{rad} / \mathrm{s})$, would require a length of only $7.2 \mathrm{~m}$ (Eq. 4). Thus, in order to achieve the required mass ratio $\mu$ (total mass of devices in relation to the mass of the structure) there would be two main problems: (i) dimensioning a pipe diameter in which there is a physically acceptable relationship between the length and diameter of the TLCD; and as a consequence, (ii) it would be necessary to install an excessive amount of dampers to achieve mass ratio values relevant for the control of structural vibration.

Before exploring the effectiveness of the MPTLCD coupled to SSI, a preliminary study of the vibration control applied only to the structure is carried out with the variation of device parameters. The efficiency of the vibration control of multiple PTLCD is analyzed for a given number of dampers, represented by the ratio $\mu$ and the influence of the ratio $\Omega$ (ratio of PTLCD frequency to structure) from changing the gas pressure of the attenuator. In this section, the base acceleration is represented by a sinusoidal function in resonance with the fundamental frequency of the structure and of amplitude $\left(\mathrm{P}_{0}\right)$ 0,2 N.

The PTLCD is tuned to the fundamental frequency of the structure with its dimensions and properties of the pressurized chamber shown in Table 8. From the analysis of the variation of the fluid coordinate $z$ in the vertical section (Figure 10), it was ensured that this displacement was compatible with the length adopted for the pressurized chamber. Such damper dimensions may appear to be overstated; however, it should be noted that the device was designed for a 40-story high-rise building.

Table 8 PTLCD dimensions and properties tuned to the fundamental frequency of the structure (considered on a fixed base).

\begin{tabular}{ccccccc}
\hline$L(m)$ & $\alpha(B / L)$ & $D(m)$ & $\mu(\%)$ & $A_{0} / A(\%)$ & $P(a t m)$ & $I_{c}(m)$ \\
\hline 40.0 & 0.90 & 1.77 & 0.25 & 12.0 & 0.622 & 2.0 \\
\hline
\end{tabular}

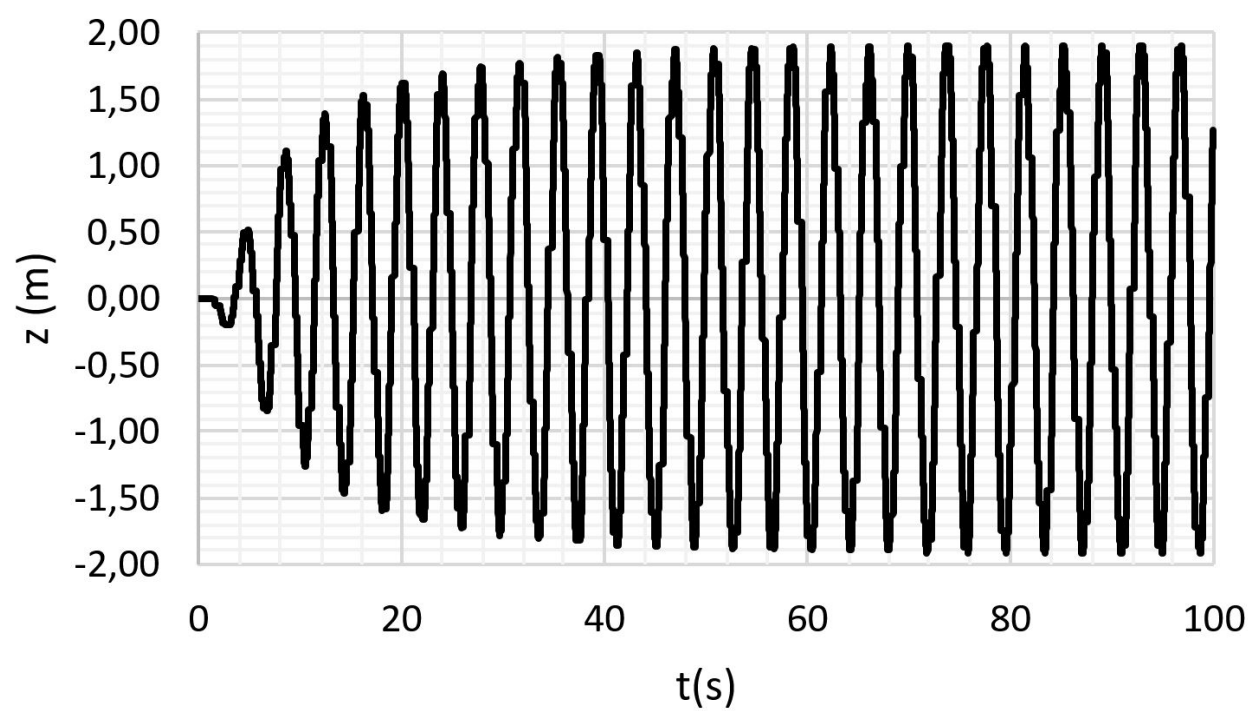

Figure 10 Fluid displacement inside the vertical section of the PTLCD.

Using this PTLCD, vibration control versus the mass ratio $\mu$ is evaluated. For each simulation the peak displacement and mean rms of the displacement during a frame of 100 seconds at the top of the structure $\left(40^{\text {th }}\right.$ story) were observed. These values are compared with the displacement at the same point of the structure, with the absence of these devices. The values obtained from the analysis are presented in Fig. 11. 

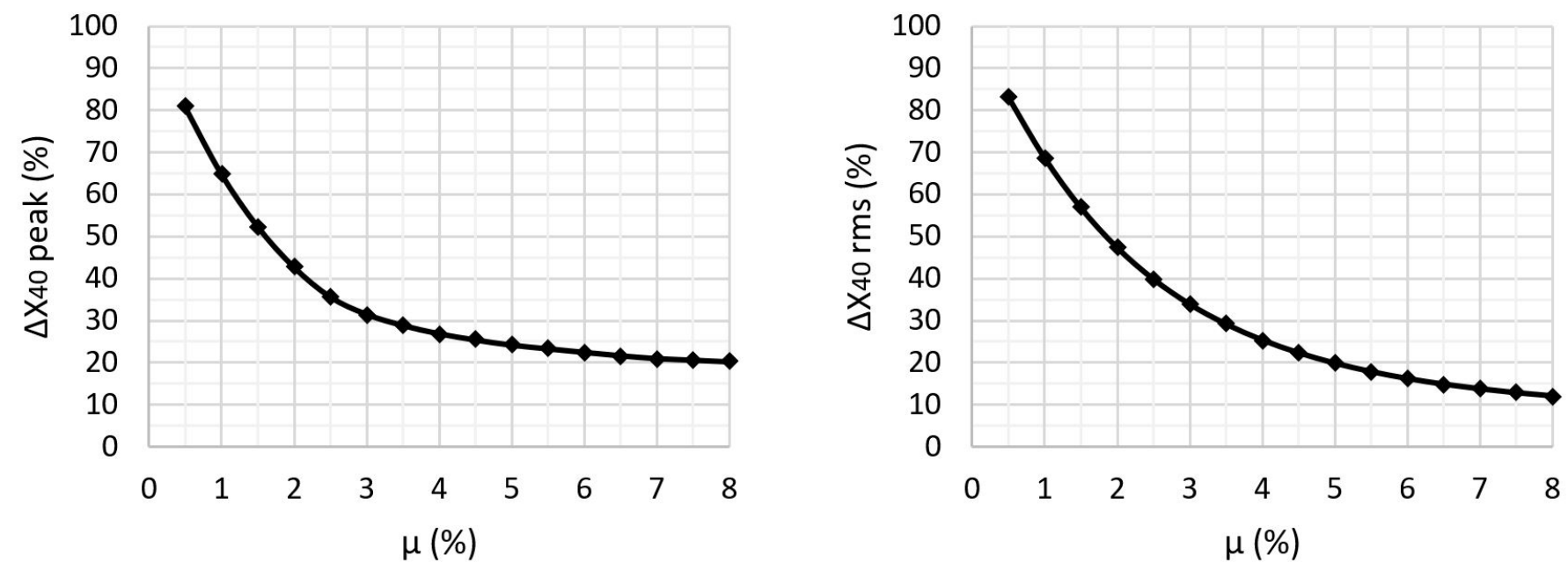

Figure 11 Variation of the peak and average rms displacement of the 40th floor versus the mass ratio $\mu$.

It can be perceived that the increase in the mass ratio (or the number) of PTLCD causes a greater reduction in the displacements of the structure and is more notable for the rms values of displacement. The peak displacement values from $\mu$ equal to $5 \%$ and beyond show an asymptotic behavior in which the difference of values from $\mu$ to $5 \%$ and to $8 \%$ is only $6 \%$ for the displacement reduction. Simmilar behavior is shown by the rms plot. The dynamic response at the top of the structure for the values of $\mu$ equal to $1 \%, 2 \%, 4 \%$ and $8 \%$ are shown in Fig. 12 . The reduction of structure displacements in the steady phase is highly sensitive to an increase of $\mu$ up to $4 \%$, and there is a slight difference from $4 \%$ to $8 \%$.

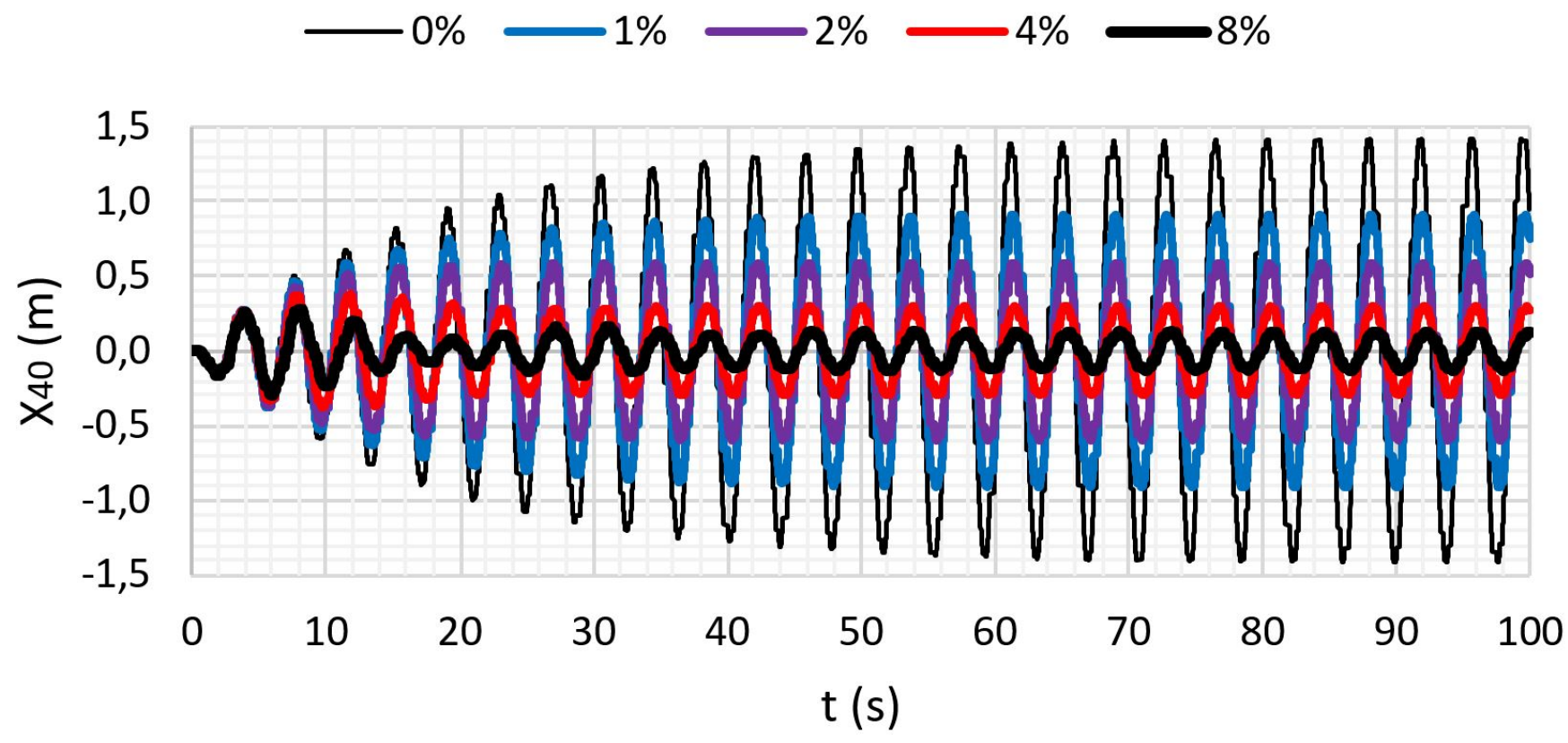

Figure 12 Displacement at the top of the structure versus the mass ratio $\mu$ for multiple PTLCD.

Another parameter of great interest for design of the PTLCD is the tunning frequency of the device. In the case of seismic excitations, the condition with the highest vibration control capability of structures is that in which the damper is in resonance with the fundamental frequency of the structure $(\Omega=1.0)$. To change the attenuator frequency, the pressure and height properties of the gas-sealed chamber are changed. The dynamic response results for $\mu$ equal to $2 \%$, $3 \%$, and $4 \%$ are shown in Fig. 13 for a variation in $\Omega$.

As shown in Fig. 12, the mass ratio $\mu$ has a great influence on vibration control. The higher the value of $\mu$, greater the efficiency in reducing the structural response. Through Fig. 13 it is also possible to state that the damper is less effective in the displacement control when shifting $\Omega$ from the fundamental frequency of the system. It is also worth noting that the greatest reduction of displacements occurs when the device is tunned with the fundamental frequency 
of the system, including the mass of the MPTLCD, and not necessarily for $\Omega=1.0$. This results from the fact that adding mass to the system (by adding dampers) and maintaining constant the stiffness of the structure leads to a reduction of the fundamental frequency of the structure-damper system. It can be observed that the minimum displacement values presented by the curve of $\mu$ equal to $3 \%$ and $4 \%$ present a shift to the left in these plots, positions where the device has a lower tuning frequency $(\Omega<1.0)$, and are in resonance with their respective structure-damper frequencies.

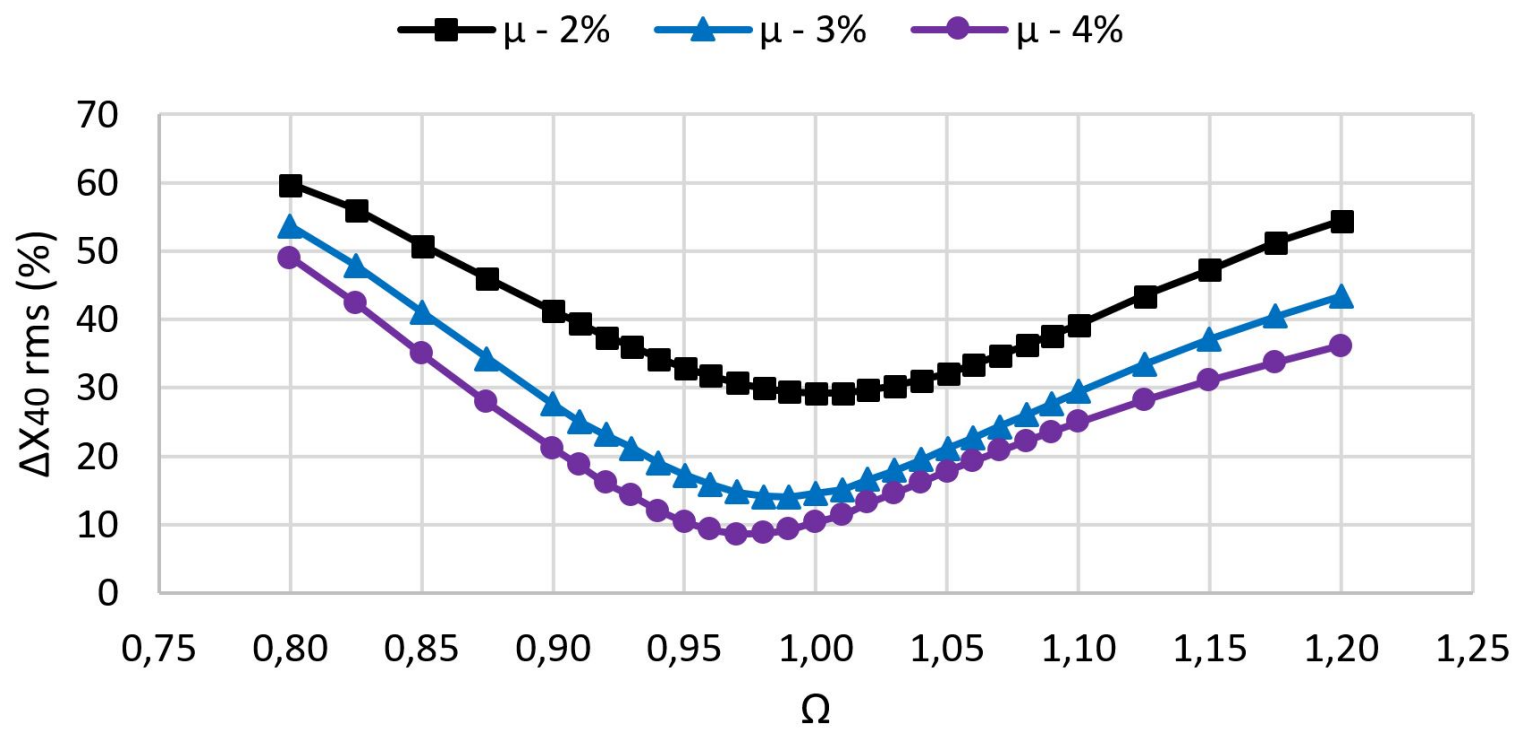

Figure 13 Variation of rms of displacements at the top of the structure with control versus the ratio between the frequency of the MPTLCDs and the fundamental frequency of the structure.

\subsection{Analysis of multiples PTLCD for flexible base structure}

After investigating the behavior of multiple PTLCD coupled to the fixed base structure and subjected to an acceleration in the base represented by a sinusoidal function, the response of the same structure is evaluated with the same control devices, but now subjected to the accelerations generated during the Kobe earthquake, as shown in Figure 9. The structural parameters of the building and soil properties are shown in Tables 3 and 4, respectively.

A series of results is presented for the vibration control of the structure versus $\mu$ for the different base properties and for MPTLCD tuned to the fundamental frequency of the structure (Figure 14); the $1^{\text {st }}$ and $2^{\text {nd }}$ natural frequency of the structure (Figure 15); and to the $1^{\text {st }}, 2^{\text {nd }}$ and $3^{\text {rd }}$ natural frequency of the structure (Figure 16). In order to tune the dampers to the $2^{\text {nd }}$ and $3^{\text {rd }}$ natural frequencies of the system, only the adjustment of the chamber pressure is done, that is, only the stiffness of the PTLCD is changed and the remaining features are kept constant (Table 8).

For the graphs in a) the dampers are tuned to the natural frequency of the fixed base structure, i.e., the tuning frequency of the control device is established without considering SSI. Although the design of the device disregards the interaction, in the forced vibration the soil-structure interaction is considered. Thus, these cases simulate the difference between theory and the application, using the theoretical scenario where vibration control neglects the effects of SSI (but in reality, the soil has an influence on the structural behavior). The graphs in b) present the results for the hypothesis that in the design of the PTLCD the natural frequencies of the structure were determined considering the SSI for each different type of soil. In summary, for tuning the device frequency, the natural frequencies of the structure considering SSI, which are shown in Table 5, were considered. The displacement values of each case, i.e., rigid base, dense soil, medium soil, and soft soil, are compared with their respective displacements shown in Table 6 for the second case, where the SSI effects are considered. 
a)
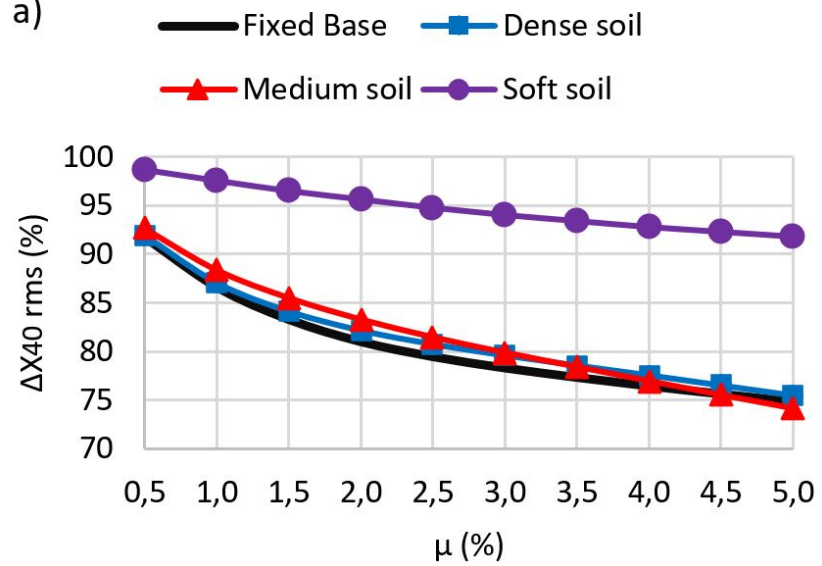

b)
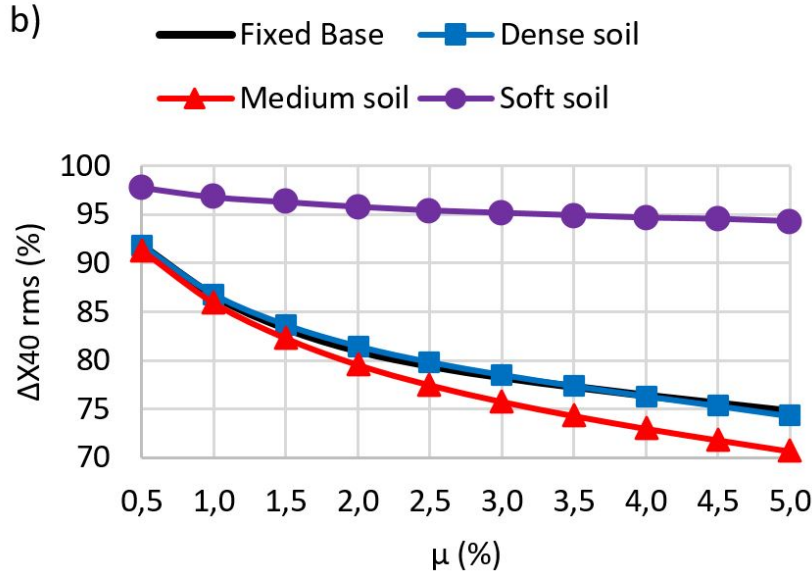

Figure 14 Rms displacement variation at the top of the frame with control versus MPTLCDs mass ratio tuned to: a) fundamental frequency of the structure under the fixed base; b) 1st fundamental frequency of the structure in flexible base.

a)

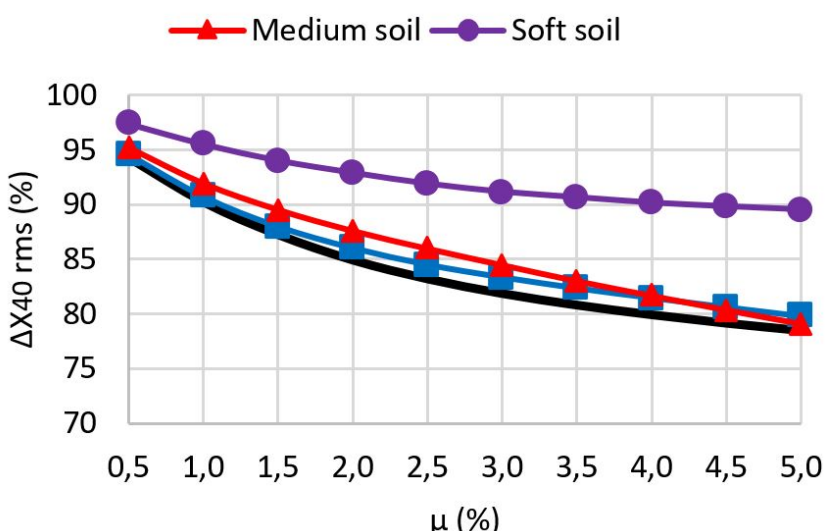

b)

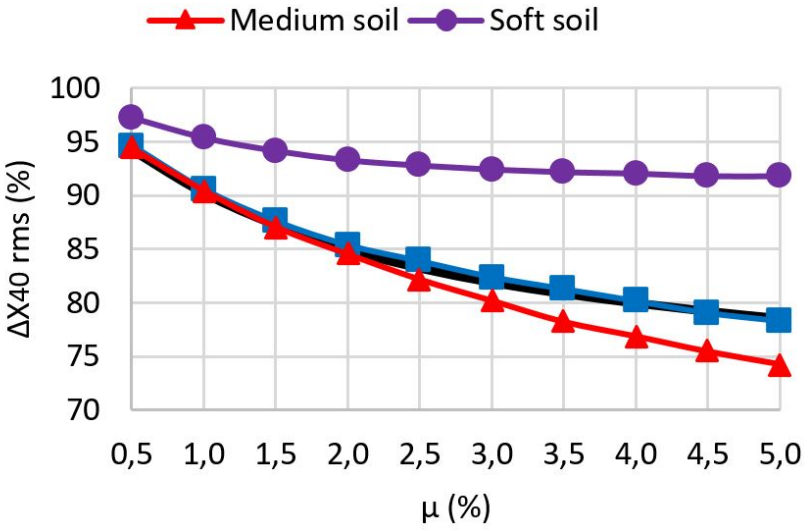

Figure 15 Rms displacement variation at the top of the frame with control versus MPTLCDs mass ratio tuned to: a) 1st and 2nd natural frequency of the structure under the fixed base; b) 1st and 2nd natural frequency of the structure in flexible base.

a)
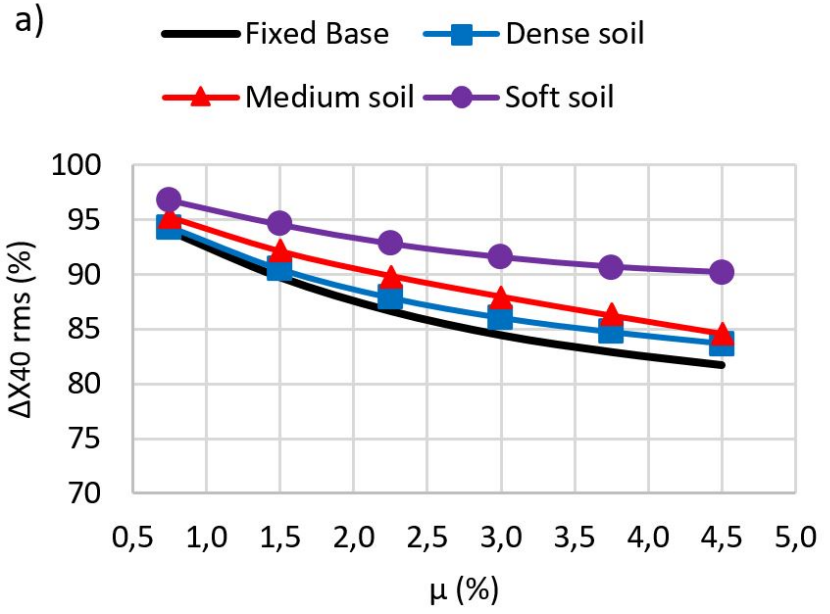

b)
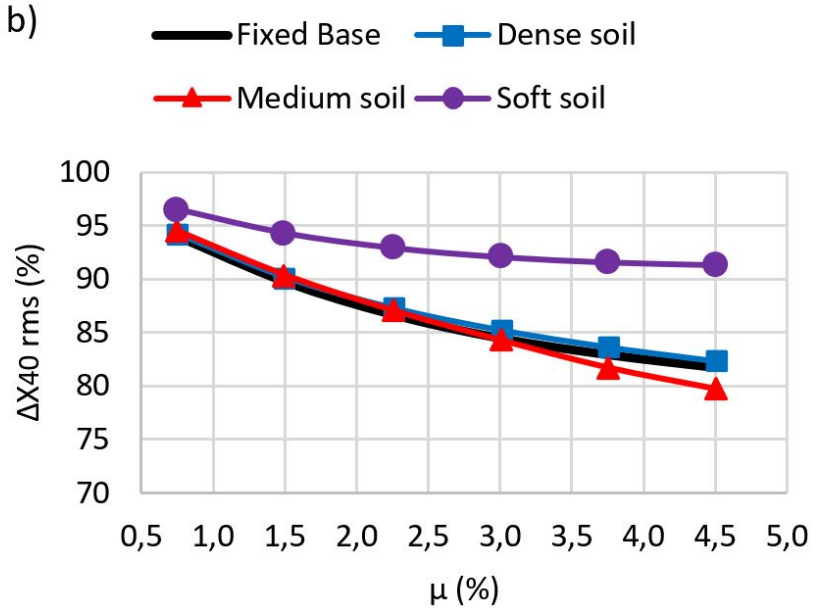

Figure 16 Rms displacement variation at the top of the frame with control versus MPTLCDs mass ratio tuned to: a) 1st, 2nd and 3rd natural frequency of the structure under the fixed base; b) 1st, 2nd and 3rd natural frequency of the structure in flexible base.

Concerning the displacements at the $40^{\text {th }}$ floor of the builing, it can be stated that the larger values of $\mu$ produce greater decreases in the displacements. Regarding the soil type, it is seen that the reduction of the maximum displacements of the structure by the PTLCD when considered the soft soil is inferior to the other models. In this case it 
is advisable to opt for another type of control device. The structure on the dense soil, which has the natural frequencies with the smallest difference to the theoretical fixed base structure, shows that SSI effects are almost negligible. Thus, it can be said that for high stiffness soils this hypothesis is legitimate. However, as described in item 4.2, when neglecting the soil-structure interaction, and specifically soil damping, an important source of energy dissipation of the system is disregarded. It can be observed that the control results for dense soil and medium soil were, respectively, slightly higher than the control for the fixed base case.

Also, under the consideration of MPTLCDs tuned to the different natural frequencies, and under the Kobe earthquake excitation, the greatest reductions of the mean displacements were obtained when the dampers were tuned solely for the $1^{\text {st }}$ natural frequency.

Table 9 contrasts the cases investigated above for MPTLCD considering the largest mass ratio and when tuned to the natural frequency of the flexible base structure (numbered from 1 to 3 and following the same order as in Figures $14 \mathrm{~b}-16 \mathrm{~b}$ ) with the results presented for the TMD to the same scenario (same structure, the same types of soil and Kobe earthquake). In general, using the vibration control with MPTLCD enables greater reductions in the response of the structure (case 1 and 2) or close values (case 3). Also, regardless of the damper model and case considered, the results obtained for the soft soil are entirely inferior to the other types of soil conditions.

Table 9 Reduction of structure displacements (40th floor) for Kobe earthquake using (considering SSI tuned dampers)

\begin{tabular}{ccccc}
\hline \multirow{2}{*}{ Device } & \multicolumn{3}{c}{ MPTLCDs } & TMD \\
\cline { 2 - 5 } & Case 1 & Case 2 & Case 3 & Farshidianfar and Soheili (2013) \\
\hline Fixed base & $25.19 \%$ & $21.50 \%$ & $18.30 \%$ & $10.90 \%$ \\
Dense soil & $25.19 \%$ & $21.68 \%$ & $17.63 \%$ & $18.38 \%$ \\
Medium soil & $29.32 \%$ & $25.72 \%$ & $20.19 \%$ & $22.31 \%$ \\
Soft soil & $5.71 \%$ & $8.20 \%$ & $8.70 \%$ & $10.13 \%$ \\
\hline
\end{tabular}

\section{CONCLUSION}

In an overview, this paper presented a formulation for the seismic analysis of structures with multiple pressurized tunned liquid column damper (MPTLCD) and with the consideration of soil-structure interaction. The computational implementation was discussed for the analysis of vibration control under different types of soils of different mechanical properties.

From the study of the soil-structure interaction it is verified that the fundamental frequency of the SSI system is lower than the fundamental frequency of the fixed-base structure, and the reductions in natural frequencies are negligible for the structure under dense soil hypothesis, but for the structure under soft soil the SSI effects are considerable (Table 5).

For the analysis of vibration control using MPTLCD, it can be observed that an increase of the mass ratio $\mu$ presents greater efficiency in the reduction of structural displacements, but there is an asymptotic behavior in this displacement reduction, in which for the analyzed cases are mostly defined for $\mu=5 \%$. On tuning the control devices, greater efficiency is achieved for vibration control when the mass of these devices is considered on the computation of the fundamental frequency.

For the scenario of MPTLCD tuned to the different natural frequencies, when the attenuators were set for the $1^{\text {st }}$ natural frequency, the largest reductions of the rms displacements were obtained under the Kobe earthquake excitation. The incorporation of pressurized chambers on the vertical sections of the " $U$ " tube presents itself as a feature that enables excellent flexibility for the dimensioning of the control device.

The authors believe that the mathematical model developed for a flexible base structure provides a better understanding of the dynamic behavior of a building with multiple PTLCD during an earthquake. The conventional procedure of tuning the attenuator to the natural frequency of the fixed base structure, i.e., fixed base, demonstrates inaccuracy for the vibration control analysis of the structure under earthquake excitation, as shown in Figs. 14-16. It is important to emphasize that SSI results show that except for the dense soil type, results can be underestimated (medium soil case) or overestimated (soft soil case) if interaction effects are neglected.

The computational routines developed in this research are available for further studies and can be easily adapted for other types of passive dampers (https://github.com/MauricioVMendes?tab=repositories). 
Author's Contributions: Conceptualization, M Mendes and L Pedroso; Formal analysis, M Mendes; Investigation, M Mendes and L Pedroso; Methodology, M Mendes, P Ribeiro and L Pedroso; Software, M Mendes; Supervision, L Pedroso; Validation, M Mendes, P Ribeiro and L Pedroso; Visualition,, M Mendes and P Ribeiro; Writing - original draft, M Mendes; Writing - original draft, M Mendes, P Ribeiro and L Pedroso.

Editor: Marcílio Alves.

\section{References}

Aghayan, A., Jaiswal, P., Siahkoohi, H. (2016). Seismic denoising using the redundant lifting scheme. Geophysics 81(3):249-260.

Banerji, P, Murudi, M. Shah, A., Popplewell, N. (2000). Tuned liquid dampers for controlling earthquake response of structures. Earthquake Engineering and Structural Dynamics 29:587-602.

Bhattacharyya, S., Ghosh, A., Basu, B. (2017). Nonlinear modeling and validation of air spring effects in a sealed tuned liquid column damper for structural control. Journal of Sound and Vibration 410:269-286.

Bigdeli, Y., Kim, D. (2016). Damping effects of the passive control devices on structural vibration control: TMD, TLD and TLCD for varying total masses, KSCE Journal of Civil Engineering 20(1):301-308.

Center For Engineering Strong Motion Data (CESMD). (2018). Accessed through the website http://www.strongmotioncenter.org

Chakraborty, S., Debbarma, R. E Marano, G. (2012). Performance of tuned liquid column dampers considering maximum liquid motion in seismic vibration control of structures. Journal of Sound and Vibration 331: 1519-1531.

Chopra, A. (1995). Dynamics of structures: theory and applications to earthquake engineering, Englewood Cliffs (New Jersey).

Dobry, R., Gazetas, G. (1986). Dynamic response of arbitrarily shaped foundations. Journal of Geotechnical Engineering 12:109-135.

Espinoza, G., Carrillo, C., Suazo, A. (2018). Analysis of a tuned liquid column damper in non-linear structures subjected to seismic excitations. Latin American Journal of Solids and Structures 15(7):e91.

Farshidianfar, A., Soheili, S. (2013). Ant colony optimization of tuned mass dampers for earthquake oscillations of high-rise structures including soil-structure interaction. Soil Dynamics and Earthquake Engineering 51:14-22.

Gao, H., Kwok, K. E Samali, B. (1999). Characteristics of multiple tuned liquid column dampers in supressing structural vibration. Engineering Structures v. 21:316-331,

Ghosh, A., Basu, B. (2004). Seismic vibration control of short period structures using the liquid column damper. Engineering Structures 26:1905-1913.

Ghosh, A., Basu, B. (2005). Effect of soil interaction on the performance of liquid dampers for seismic applications. Earthquake Engineerign and Structural Dynamics 34:1375-1389.

Hartog, D. (1956). Mechanical vibrations, McGraw-Hill (New York).

Hochrainer, M., Ziegler, F. (2006). Control of tall building vibrations by sealed tuned liquid column dampers. Structural Control and Health Monitoring 13:980-1002.

Hokmabady, H., Mohammadyzadeh, S., Mojtahedi, A. (2019). Suppressing structural vibration of jacket-type platform employing a novel Magneto-Rheological Tuned Liquid Column Gas Damper. Ocean Engineering 180:60-70.

Jabary, R., Madabhushi, G. (2018).Tuned mass damper positioning effects on the seismic response of a soil-MDOF-structure system. Journal of Earthquake Engineering 22 (8):1317-1335.

Lee, S., Min, K., Lee, H. (2011). Parameter identification of new bidirectional tuned liquid column and sloshing dampers. Journal of Sound and Vibration 330:1312-1327.

Liu, M., Chiang, W., Hwang, J., Chu, C. (2008). Wind-induced vibration of high-rise building with tuned mass damper including soil-structure interaction. Journal of Wind Engineering and Industrial Aerodynamics 96:1092-1102. 
Luco, J. (1998). A simple model for structural control including soil-structure interaction effects. Earthquake Engineering and Structural Dynamics 27:225-242.

Marano, G., Quaranta, G., Sgobba, S. (2010). Fuzzy-entropy based robust optimzation criteria for tuned mass dampers. Earthquake Engineering and Engineering Vibration 9(2):285-294.

Mendes, M. (2018). Seismic analysis of buildings with soil-structure interaction and pressurized liquid column dampers. M.Sc. Dissertation, Brasília University, Brazil.

Min, K., Kim, H., Lee, S., Kim, H., Ahn, S. (2005). Performance evaluation of tuned liquid column dampers for response control of a 76-story benchmark building. Engineering Structures 27:1101-1112.

Mousavi, S., Bargi, K., Zahrai, S. (2013).Optimum parameters of tuned liquid column-gas damper for mitigation of seismicinduced vibrations of offshore jacket platforms. Structural Control and Health Monitoring 20:422-444

Mylonakis, G., Nikolau, S., Gazetas, G. (2006). Footings under seismic loading: analysis and design issues with emphasis on bridge foundations. Soil Dynamics and Earthquake Engineering 26:824-853.

Newmark, N. (1959). A method of computation for structural dynamics. Journal of Engineeng Mechanics 85(3):67-94.

Ozuygur, A., Gunduz, A. (2018). Optimal control of structures under earthquake including soil-structure interaction. Journal of Earthquake Engineering 22(8):1317-1335.

Pais, A., Kausel, E. (1988). Approximate formulas for dynamic stiffness of rigid foundations. Soil Dynamics and Earthquake Engineering 7: 213-227.

Pedroso, L. (1992). Mechanical analogy for a study of an incompressible fluid oscillating column with stiffness and dissipation effects. Techincal Report, Brasilia University, Brazil.

Rozas, L., Boroschek, R., Tamburrino, A., Rojas, M. (2016). A bidirectional tuned liquid column damper for reducing the seismic response of buildings. Structural Control and Health Monitoring 23:621-640.

Shahrabi, M., Bargi, K. (2019). Enhancement the fatigue life of a floating breakwater mooring system using tuned liquid column damper. Latin American Journal of Solids and Structures 16(7):e220.

Shum, K., Xu, Y. (2004). Multiple tuned liquid column dampers for reducing coupled lateral and torsional vibration of structures. Journal of Wind Engineering and Industrial Aerodynamics 26:745-758.

Shum, K., Xu, Y., Guo, W. (2008). Wind-induced vibration control of long span cable-stayed bridges using multiple pressurized tuned liquid column dampers. Engineering Structures 96:166-192.

Sonmez, E., Nagarajaiah, S., Sun, C., Basu, B. (2016). A study on semi-active Tuned Liquid Column Dampers (sTLCDs) for structural response under random excitations. Journal of Sound and Vibration 362:1-15.

Souza, R. (2003). Passive/active control of oscillations of slender structures by means of dynamic fluid devices. Ph.D. Thesis, Rio de Janeiro Federal University, Brazil.

Tait, M. (2008). Modelling and preliminary design of a structure-TLD system. Engineering Structures 30:2644-2655.

Warbuton, G. (1981). Optimum absorber parameters for minimizing vibration response. Earthquake Engineering and Structural Dynamics 9:251-262.

Warbuton, G. (1982). Optimum absorber parameters for various combinations of response and excitation parameters. Earthquake Engineering and Structural Dynamics 10:381-401. 